\title{
Interpreting the galaxy group CG J1720-67.8 through evolutionary synthesis models
}

\author{
S. Temporin ${ }^{1, \star}$ and U. Fritze-von Alvensleben ${ }^{2}$ \\ ${ }^{1}$ Institut für Astrophysik, Leopold-Franzens-Universität Innsbruck, Technikerstraße 25, 6020 Innsbruck, Austria \\ e-mail: giovanna. temporin@uibk.ac . at \\ 2 Universitätssternwarte, Geismarlandstr. 11, 37083 Göttingen, Germany \\ e-mail: ufritze@uni-sw.gwdg.de
}

Received 5 June 2005 / Accepted 22 September 2005

\section{ABSTRACT}

This paper is part of a series devoted to a detailed analysis of the properties of the compact group CG J1720-67.8 and its member galaxies with the aim of shedding light on its evolutionary history. Here we interpret our previously published observational results through comparison with chemically consistent spectrophotometric evolutionary synthesis models to gain further clues to the evolution of the galaxies in this group. In order to reduce the number of free parameters, we considered the simplest case of a single burst of star-formation turned on after 11-12 Gyr of undisturbed galaxy evolution. However, we also briefly explored the effect of multiple, interaction-induced bursts of star-formation. We found that the two spiral galaxies are consistent with interaction-induced strong starbursts switched on 40 to 180 Myr ago and still active. For the early-type galaxy, a $\lessgtr 0.9-1.3 \mathrm{Gyr}$ old star-formation event (depending on the considered model) appears consistent with the observed properties. The comparison with models cannot rule out the possibility that this galaxy is already the result of a merger. Alternatively, a starformation episode in this galaxy might have been triggered by gas inflow as a consequence of the interaction with the companion galaxies. Estimates of galaxy masses were derived from the comparison with the models. Finally our results are discussed in comparison with other well-studied, poor galaxy systems.

Key words. galaxies: evolution - galaxies: interactions - galaxies: starburst

\section{Introduction}

Hierarchical evolution theories come in different flavors depending on the assumed cosmologies (e.g. Lacey \& Cole 1993; Kauffmann et al. 1999; Cole et al. 2000; Springel et al. 2005); however, they all suggest that at least part of the existing elliptical galaxies are the products of galaxy mergers. Such merging phenomena are thought to be particularly effective in compact galaxy groups (CGs) today. These are, in fact, dense environments where the low velocity dispersion of the galaxies - comparable to their rotational velocities - and their small mutual distances imply considerably short crossing times. Tidal friction can slow the groups' members down, thereby allowing them to merge, although the CGs' dark matter content and distribution might play fundamental roles in their evolution (Athanassoula et al. 1997). Also, according to hierarchical models of structure formation, groups are to be considered the building blocks of clusters; therefore, understanding the evolution of galaxies in the group environment is useful for interpreting the galaxy population observed in clusters.

* Present address: INAF - Osservatorio Astronomico di Brera, Via Brera 28, 20121 Milano, Italy.
X-ray studies have given important support to the idea that the final fate of CGs is their coalescence into bright field elliptical galaxies, in some of which possible CGs relics have been identified (e.g. Ponman et al. 1994; Mulchaey \& Zabludoff 1999; Vikhlinin et al. 1999). Miles et al. (2004) indicate X-ray dim or low-velocity dispersion groups as present sites of rapid dynamical evolution and possible modern precursors of fossil groups. Nevertheless, there is no lack of challenges to this apparently simple scenario. In fact, recent studies of stellar populations of galaxies in compact groups point to old ages for their elliptical members (Mendes de Oliveira et al. 2005a; Proctor et al. 2004), a finding at odds with the idea that ellipticals might have formed through subsequent mergers in the dense group environment. Obviously, the question of how groups and galaxies within groups evolve has not been settled yet. Investigations of groups that appear to be in a merging phase should help for understanding these evolutionary processes. Nearby systems in the merging phase are rarely observed, but are best-suited to detailed studies that might serve as benchmarks in the interpretation of more distant galaxy systems. This work deals with the interpretation of such a rare example of a galaxy group caught shortly before final merging. 
CG J1720-67.8 is a complex galaxy system with various signs of ongoing and past interactions. The group is composed of three main galaxies and a number of candidate tidal dwarf galaxies in an extremely dense spatial configuration with low velocity dispersion (Weinberger et al. 1999, hereafter Paper I). This peculiar galaxy group is representative of the last phases of $\mathrm{CG}$ evolution leading to the coalescence of the brightest members. The rarity of nearby systems in such a configuration is made evident by the fact that the only other example of CG found in a similar evolutionary phase is HCG 31 (Amram et al. 2004). In a series of papers devoted to a detailed observational study of CG J1720-67.8 (Temporin et al. 2003a,b, 2005a, hereafter Paper II, III, and IV, respectively), we presented the optical photometry and spectroscopy of all group members, radio continuum and $\mathrm{H}$ I observations, and farinfrared emission of the group. We analyzed the physical and chemical properties of the ionized gas by use of the observed emission-lines and CLOUDY (Ferland 1996) photoionization models and investigated the ionized gas velocity field. In the light of these observations, we discussed the star-formation properties of the system and suggested some possible interaction scenarios that might have led to the present group configuration (Paper IV). Here we concentrate on the three main galaxies and used chemically consistent, spectrophotometric evolutionary synthesis models to obtain the simplest possible interpretation of the observed properties and check their consistency with the interaction history proposed in Paper IV. In particular, we aim at estimates of the ages and strengths of the most recent star-formation episodes, which have most likely been triggered by galaxy interactions within the group. For our purpose, we use the lowest number of free parameters that provides reasonable agreement with the available observations. For the comparisons with models, we then make use of the observational data and derived quantities (such as star-formation rates, metallicities, extinction properties, luminosities) that are extensively discussed in Papers I to IV. We refer the reader to those papers for detailed information. Here we only briefly describe the available datasets and summarize the main properties of the galaxies (Sect. 2.1), which will be useful for the goals of this work. We also recall the basics and input physics of the evolutionary synthesis code GALEV (Schulz et al. 2002; Anders \& Fritze-v. Alvensleben 2003; Bicker et al. 2004), which we use for our models (Sect. 2.2). In Sects. 3 and 4 we present the simplest models that reasonably match the observations for the late-type and early-type galaxies, respectively. In Sect. 5 we investigate the possibility of multiple bursts of star-formation and, finally, in Sect. 6 discuss our results in the framework of the possible interaction history of the group.

\section{Data and methodology}

\subsection{Observational background}

Photometric and spectroscopic data were obtained at the ESO $2.2 \mathrm{~m}$ and $3.6 \mathrm{~m}$ telescopes (long-slit and multiobject spectroscopy, $B V R$ imaging), in Las Campanas at the Du Pont $2.5 \mathrm{~m}$, Swope $1 \mathrm{~m}$, and Magellan $6.5 \mathrm{~m}$ telescopes (long-slit spectra and $J H K_{\mathrm{s}}$ imaging), and at the
Anglo-Australian Observatory AAT $3.9 \mathrm{~m}$ telescope (mosaic of integral field spectra including $\mathrm{H} \alpha$, [N II], [S II] emission lines and adjacent continuum). Observations in the $\mathrm{HI} 21 \mathrm{~cm}$ line and in the radio continuum at 6,13 , and $20 \mathrm{~cm}$ were obtained at the Australia Telescope Compact Array.

The three main galaxies of CG J1720-67.8, numbered 1, 2, and 4 in Papers I through IV (G1, G2, and G4, hereafter), are classified as an Scd, an S0, and an Sc galaxy, respectively, on the basis of the color analysis and a quantitative morphological study, although the strong morphological disturbances caused by their mutual interactions made it difficult to classify these galaxies within traditional schemes (Fig. 1). Where G2 shows a typical $r^{1 / 4}$ bulge, G1 and G4 have exponential bulges.

The three galaxies, as well as the candidate tidal dwarf galaxies, exhibit emission line spectra typical of $\mathrm{HII}$ or starburst galaxies. The $\mathrm{H} \alpha$ emission indicates considerable star-formation activity in the two spirals and a small amount of star-formation that is essentially confined to the central part of the $\mathrm{S} 0$ within a radius of $\approx 1.2 \mathrm{kpc}$. Indeed, the star-formation rate densities (SFRDs) derived from the $\mathrm{H} \alpha$ emission are comparable to and, in some cases, higher than those typical of interacting galaxies (Papers I and II).

Both the enhanced star-formation activity in their nuclei and throughout their disks and the clumpy distribution of star-forming regions of G4 appear evident on the $\mathrm{H} \alpha$ map (Paper IV). In fact, the high bulge-to-total-light ratio $B / T>0.4$ of $\mathrm{G} 1$ could be a consequence of the central star-formation, which might mimic the presence of an important bulge in an otherwise apparent late-type galaxy.

Total SFRs for the two galaxies can be derived from their $\mathrm{H} \alpha$ luminosities, as obtained in Paper IV (Table 5) from integral field spectroscopic observations, after correction for internal extinction. An average value of the extinction in the emission lines, as given by the Balmer decrement in case B approximation, is evaluated to be $E(B-V)_{\mathrm{g}} \approx 0.5 \mathrm{mag}$ for $\mathrm{G} 1$ (from long-slit spectra, see Paper II) and $\approx 0.6 \mathrm{mag}$ for $\mathrm{G} 4$ (from integral field spectra, Paper IV). Total SFRs calculated following Kennicutt (1998a) are $\sim 2.8$ and $\sim 3.2 M_{\odot} \mathrm{yr}^{-1}$ for G1 and $\mathrm{G} 4$, respectively.

We do not have information on the distribution of extinction in G1, but for G4 we found evidence of a patchy distribution of dust. Indeed, the extinction map obtained in Paper IV shows regions with extinction well in excess of the average value used for the correction. Therefore, the estimated SFR of G4 is likely to be only a lower limit to the actual SFR. This hypothesis is supported by the higher total SFR of the group obtained from extinction-unaffected radio continuum emission, $S F R_{1.4 \mathrm{GHz}}=18 M_{\odot} \mathrm{yr}^{-1}$, with respect to the one based on $\mathrm{H} \alpha$ emission, $S F R_{\mathrm{H} \alpha}=10 M_{\odot} \mathrm{yr}^{-1}$. Then G4 contributes a third of the total observed $\mathrm{H} \alpha$ emission, but probably more than a third of the $1.4 \mathrm{GHz}$ continuum emission, since the extended radio source is peaked at G4. We therefore expect its actual $S F R$ to be $\gtrsim 6 M_{\odot} \mathrm{yr}^{-1}$.

The above arguments must be considered when comparing the observed SFR with values given by the evolutionary synthesis models. The integrated $\mathrm{H} \alpha$ emission-line flux of G2 (see Paper IV, Tables 1 and 5), when corrected for internal 


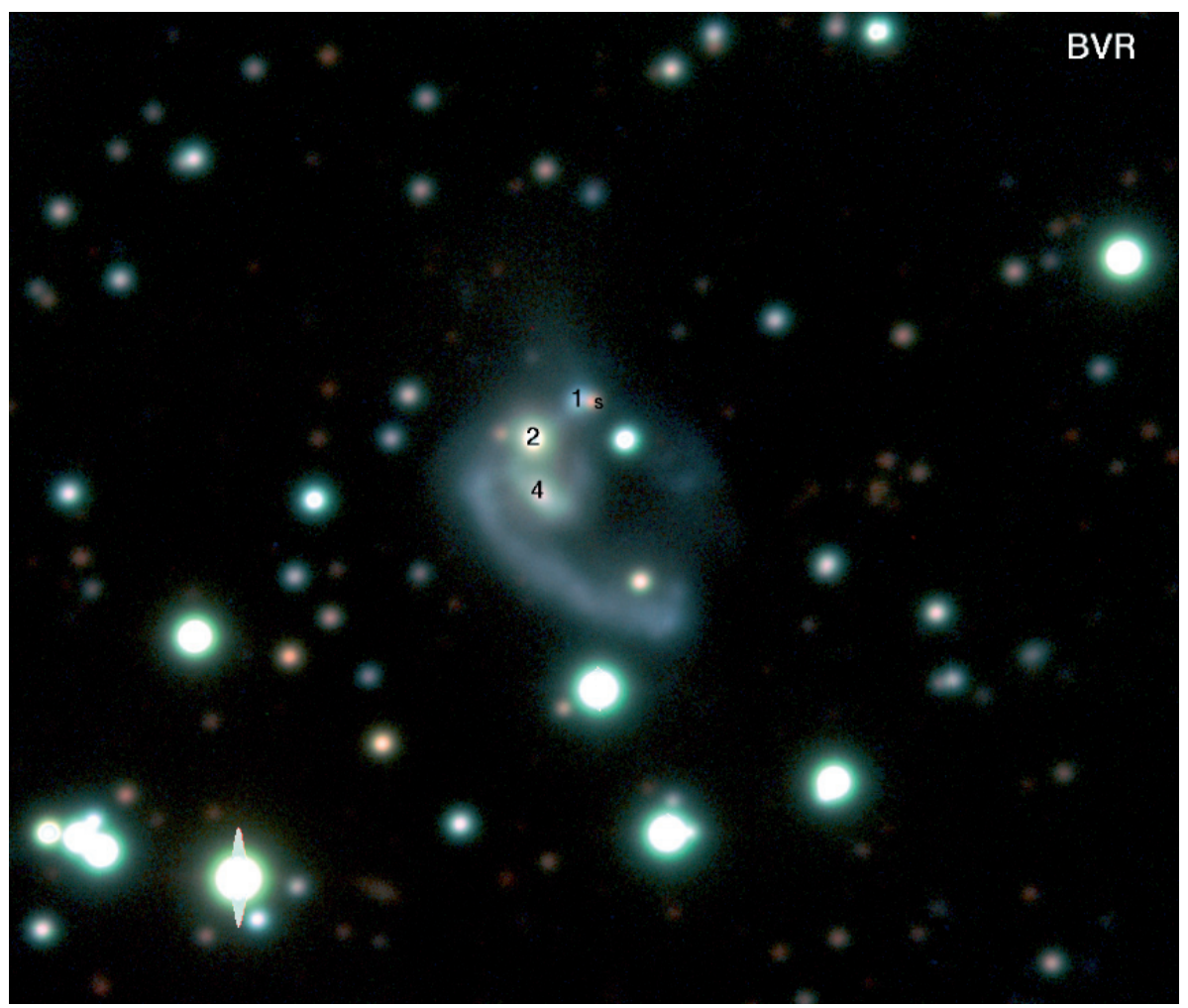

Fig. 1. $B V R$ composite image of CG J1720-67.8. Numbers indicate the three main galaxies, while the "s" label indicates an M-type foreground star seen in projection on G1. North is on top, east to the left. The size of the field is $\sim 2 ! 3 \times 1 ! 9$. At the distance of the group, $1^{\prime \prime} \sim 0.9 \mathrm{kpc}$.

extinction, implies an $S F R^{1} \sim 0.4$ to $0.8 M_{\odot} \mathrm{yr}^{-1}$. The three galaxies appear in mutual interaction, and a bright, knotty tidal tail departing from $\mathrm{G} 4$ extends for $\approx 29 \mathrm{kpc}\left(H_{0}=\right.$ $\left.75 \mathrm{~km} \mathrm{~s}^{-1} \mathrm{Mpc}^{-1}\right)$. From the maximum amplitude of the velocity curve of G4 and the projected length of the tidal tail, we estimated for it an age of $\approx 200 \mathrm{Myr}$ (Paper IV). All the group members show blue colors, with the exception of G2 whose observed red color $(B-V=0.98)$ is probably a combined effect of the high internal reddening $\left(A_{\mathrm{V}}=1.6 \mathrm{mag}\right.$, as derived from the Balmer decrement in its spectrum) and of its metallicity $\left(Z \sim 0.5 Z_{\odot}\right.$, based on emission line measurements), higher than that of all other group members, which have $Z \sim 0.3 Z_{\odot}$ or lower (see Papers II and III).

\subsection{Evolutionary synthesis with the code GALEV}

We used the Göttingen evolutionary synthesis code GALEV as first presented by Fritze-v. Alvensleben \& Gerhard (1994), improved/updated by Kurth et al. (1999) and then by Schulz et al. (2002). The version of the code we applied is based on the Padova isochrones for metallicities ${ }^{2}$ in the range $10^{-4} \leq$ $Z \leq 0.05$ (Bertelli et al. 1994, as obtained from the Padova web server in the extended and improved version of November 1999), extended toward lower stellar masses $\left(0.15-0.45 M_{\odot}\right)$ by zero-age main sequence points from Chabrier \& Baraffe (1997). A complete library of model atmosphere spectra - in

\footnotetext{
${ }^{1}$ Uncorrected values would be 0.1 to $0.2 M_{\odot} \mathrm{yr}^{-1}$.

${ }^{2} Z$ is the abundance by mass of elements that are more massive than ${ }^{4} \mathrm{He} ; Z_{\odot}=0.018$.
}

terms of spectral types from hottest to coolest stars and all luminosity classes - from Lejeune et al. $(1997,1998)$ was implemented for the full range of metallicities spanned by the isochrones. Spectra of all states along an isochrone for a given metallicity are summed up, since this is a one-zone model without any spatial resolution, and weighted with the initial mass function (IMF). Two different IMFs were available, a Salpeter (1955) IMF and a Scalo (1986) IMF, the latter parametrized as $\phi(m) \sim m^{-x}$ with $x=-1.25$ for $m_{1} \leq m \leq 1 M_{\odot}$, $x=-2.35$ for $1 M_{\odot} \leq m \leq 2 M_{\odot}$, and $x=-3.00$ for $2 M_{\odot} \leq m \leq m_{\mathrm{u}}$. For both IMFs the lower and upper mass limits $m_{1}$ and $m_{\mathrm{u}}$ are $0.08 M_{\odot}$ and $85 M_{\odot}$, respectively. In our models we adopted the Scalo IMF and assumed a fraction of visible mass $F V M=0.5$, which gives mass-to-light ratios for undisturbed galaxies after $\sim 12 \mathrm{Gyr}$ in agreement with observations and implies that $50 \%$ of the mass transformed into stars is in the form of Jupiter-like objects or brown dwarfs (Fritze-v. Alvensleben \& Gerhard 1994).

Nebular emission is not included in the version of the code we used; however, the influence of nebular emission on colors has been evaluated by Anders \& Fritze-v. Alvensleben (2003) using an updated version of GALEV. In particular, the impact of gaseous emission on broad-band colors is found to be negligible, except for very strong bursts in very early stages: ages < few $10^{6}$ yr up to $\sim 2 \times 10^{7} \mathrm{yr}$, slightly depending on metallicity. Although GALEV allows for evolutionary and cosmological corrections, including attenuation effects by intergalactic hydrogen, we did not apply them, since they are unimportant at the relatively low redshift $(z=0.045)$ of CG J1720-67.8. 
The model calculations start with a proto-galaxy of given total mass that is maintained constant during the whole evolution (closed-box model). The metallicity $Z$ of the stellar population can be chosen among five different values $(0.0004,0.004$, $0.008,0.02$, and 0.05 ) or calculated in a chemically consistent (CC) way, i.e. accounting for the increasing initial metallicity of successive generations of stars (Fritze-v. Alvensleben 1999).

In the presence of starburst-driven galactic scale winds or gas accretion events, the closed-box representation might be inadequate, so in such a situation it could be useful to assume a fixed metallicity (i.e. non-CC model) rather than to introduce an exceedingly high number of additional free parameters in a $\mathrm{CC}$ model (e.g. in/outflow rate, timescale, metallicity, and time evolution).

In both non- $\mathrm{CC}$ and $\mathrm{CC}$ models, the initial abundance in the gas is set to $Z(0)=10^{-4}$. Chemical composition gradients in the galaxy are neglected. The increase in gas metallicity with time and the gas content are calculated by solving a modified form of Tinsley's equations (Tinsley 1980) with stellar yields for type I and II supernovae, planetary nebulae, and stellar mass loss (Lindner et al. 1999).

The composite stellar population of a galaxy is obtained by folding models for single stellar populations with a particular star-formation history (SFH). Different SFHs are adopted appropriately for different spectral galaxy types. An exponential parametrization of the star-formation rate (SFR), $\Psi(t) ~ ~$ $\exp \left(-t / t_{*}\right)$ is used for elliptical galaxies, while a linear function of the gas-to-total mass ratio, $\Psi(t) \sim \frac{\mathrm{G}}{\mathrm{M}}(t)$, is assumed for Sa to Sc spirals (according to what was found by e.g. Kennicutt 1998b). The constant of proportionality (i.e. the efficiency factor) is chosen to yield characteristic gas consumption timescales for the respective spiral types and decreases going from S0 to late-type spirals. Except for the fact that some gas is given back as soon as the first stars die, this is similar to the timescale for the decrease of the SFR from its initial value. This initial SFR is highest for ellipticals and becomes progressively lower for later types. A constant SFR is assumed for Sd galaxies.

Characteristic time scales for star-formation are $1 \mathrm{Gyr}$ for ellipticals and 2, 3, 10, and $16 \mathrm{Gyr}$ for $\mathrm{Sa}, \mathrm{Sb}, \mathrm{Sc}$, and Sd galaxies, respectively. Several quantities are calculated on the basis of these SFHs at every timestep of the galaxy evolution: luminosities, color indices, star and gas masses, metallicities, ejection rates of gas and metals by dying stars, SFR, and the spectra in the wavelength range from UV to NIR. The SFHs are chosen so that the colors, spectra, and gas content of the model galaxies after $12 \mathrm{Gyr}$ of undisturbed evolution are brought into agreement with the average observed colors, template spectra, and typical gas fraction of the respective galaxy types in the RC3 catalog (Buta et al. 1995; Buta \& Williams 1995; Kennicutt 1992). Galaxy luminosities in $B$ are normalized to the average observed $B$-band luminosity $\left\langle M_{B}\right\rangle$ of the respective galaxy types in Virgo cluster (Sandage et al. 1985) after $12 \mathrm{Gyr}$ of evolution. More detailed explanations, as well as galaxy model spectra for various spectral types and ages in the range $4 \mathrm{Myr}$ to $15 \mathrm{Gyr}$, are presented by Bicker et al. (2004).
On top of the normal evolution, one or more bursts of star-formation can be implemented. A starburst is simulated by adding to the model an exponential SFR with an e-folding timescale $t_{*}$ of about $10^{8} \mathrm{yr}$. The maximum burst strength is governed by the gas reservoir of the galaxy at the time of the onset of the burst. The burst strength is quantified by the parameter $b$ defined as the ratio of the total stellar mass formed during the burst (i.e. integrated until the time at which the burst is exhausted, the residual SFR is too low to balance the return of mass to the gas reservoir through stellar winds, and the total stellar mass does not increase any further) to the mass of stars present in the galaxy at the onset of the burst.

\section{Late-type group members: G1 and G4}

Our analysis in Paper II showed that G1 and G4 have photometric and spectroscopic characteristics that are consistent with Scd and Sc morphological types, respectively, although none of these galaxies shows clear spiral arms, and G1 has a high bulge-to-total light ratio. We interpret these morphological characteristics as indicating an undergoing transition of the galaxies toward earlier morphological types as an effect of their interactions. Bulge-to-disk ratios and spectral types can be affected by strong bursts, so that caution is necessary when choosing the SFH to be adopted in the models. Given the above considerations, we judged it reasonable to assume SFHs that are typical of late-type spirals, i.e. $\mathrm{Sd}$ or $\mathrm{Sc}$, when modeling $\mathrm{G} 1$ and G4. Since modeling attempts with an Sd-type SFH did not produce satisfactory results ${ }^{3}$, we present here only models that assume an Sc-type SFH.

In our models we simulated undisturbed evolution of an Sc galaxy for 12 Gyr (which accounts for the older stellar populations of the galaxies), then added a starburst event on top of the normal evolution. The color evolution of the model after the onset of the burst, calculated with a timestep of $4 \mathrm{Myr}$, was compared with optical/NIR observed colors of the galaxies to obtain a first rough indication of the burst strength and age. The timescale of the starburst should be on the order of a dynamical timescale in the burst region. For a given burst strength, changing the timescale of the burst would not change the shape of the optical spectrum remarkably, so it would have little influence on the determination of the burst age, while it would affect the luminosity at a given age, thus influencing the final estimate of the total mass of the galaxy. In our models we assumed a burst timescale of $2 \times 10^{8} \mathrm{yr}$. Models with timescale $1 \times 10^{8} \mathrm{yr}$ would lead to the same burst ages estimated below and, for the ages of interest, would give differences in absolute magnitudes in the range 0.01-0.05 mag and would imply lower total masses of the galaxies by a factor $\approx 1.2$.

\footnotetext{
${ }^{3}$ Models that assume an Sd-type SFH produce similar results to those presented in this section for the Sc-type SFH concerning the burst strengths, the best-fit burst ages, the best-fit internal extinctions, and the mass estimates for G1 and G4; however, they predict values that are too high for SFRs and metallicities with respect to the observations and, at the best-fit ages, they disagree with the central part of the observed optical spectra and with the observed optical-NIR spectral energy distribution. Disagreements are more significant for G4 than for G1, as expected from their morphological classification.
} 
Table 1. G1 and G4: observed ${ }^{a}$ and modeled colors.

\begin{tabular}{lllllllll}
\hline \hline Object/Model & $B-V$ & $V-R$ & $V-K$ & $B-R$ & $B-H$ & $J-H$ & $H-K$ & $Z / Z_{\odot}$ \\
\hline G1 & 0.47 & 0.30 & 2.43 & 0.78 & 2.81 & 0.43 & 0.09 & $\sim 0.2$ \\
G4 & 0.55 & 0.51 & 2.20 & 1.06 & 2.57 & 0.48 & 0.18 & $\sim 0.1$ \\
& & & & & & & & \\
CC-Sc, $b=0.5,40 \mathrm{Myr}$ & 0.26 & 0.35 & 1.91 & 0.61 & 1.98 & 0.49 & 0.19 & 0.22 \\
CC-Sc, $b=0.5,180 \mathrm{Myr}$ & 0.23 & 0.31 & 1.97 & 0.54 & 1.99 & 0.53 & 0.21 & 0.30 \\
CC-Sc, $b=0.5,320 \mathrm{Myr}$ & 0.27 & 0.34 & 2.11 & 0.60 & 2.15 & 0.55 & 0.23 & 0.37 \\
& & & & & & & & \\
CC-Sc, $b=0.25,40 \mathrm{Myr}$ & 0.36 & 0.41 & 2.08 & 0.77 & 2.25 & 0.51 & 0.20 & 0.21 \\
CC-Sc, $b=0.25,180 \mathrm{Myr}$ & 0.32 & 0.37 & 2.08 & 0.69 & 2.19 & 0.53 & 0.21 & 0.24 \\
CC-Sc, $b=0.25,320 \mathrm{Myr}$ & 0.35 & 0.39 & 2.18 & 0.74 & 2.31 & 0.55 & 0.22 & 0.26 \\
\hline
\end{tabular}

${ }^{a}$ Observed colors after the application of a standard inclination correction.
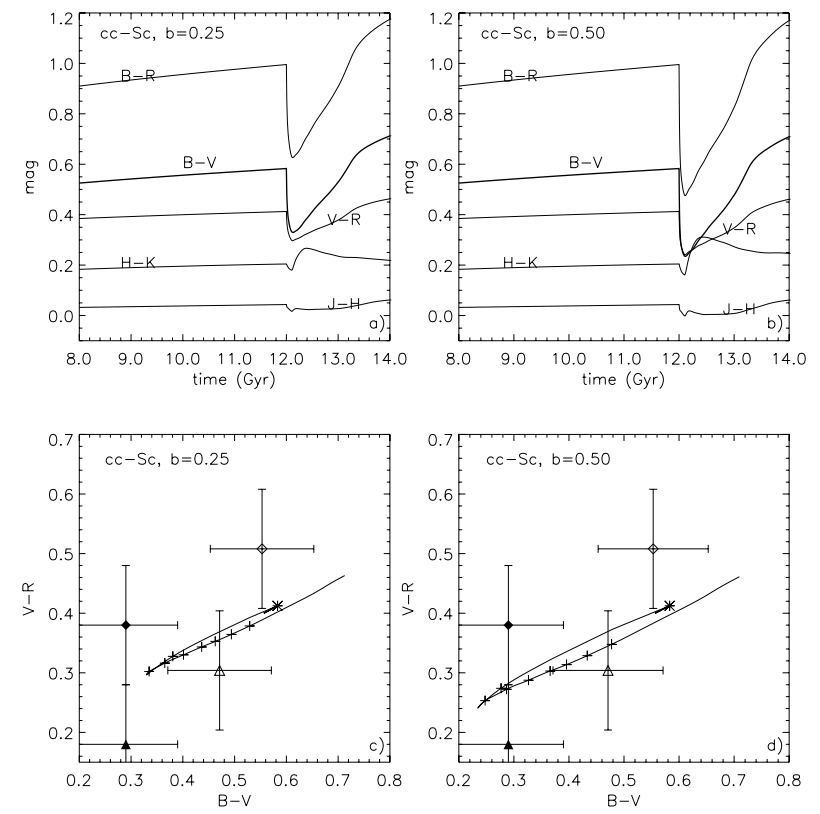

Fig. 2. a) Evolution of color indices for a cc-Sc model with a $25 \%$ burst switched on after $12 \mathrm{Gyr}$ of undisturbed evolution. The $B-V$ index is indicated with a thick line to avoid confusion with other indices. For the same reason, an offset of -0.5 has been applied to the $J-H$ index. b) Same as a), for a $50 \%$ burst. c) Color-color diagram showing the color indices evolution during the burst phase for a cc-Sc model with a $25 \%$ burst. The asterisk marks the onset of the burst at a galaxy age of 12 Gyr. Plus symbols are plotted for burst ages from $40 \mathrm{Myr}$ to $1 \mathrm{Gyr}$ at intervals of $140 \mathrm{Myr}$. The empty triangle and diamond indicate the observed colors of G1 and G4, respectively, after a standard inclination correction. The full triangle and diamond indicate the observed colors after correction for internal extinction using $E(B-V)=0.5 \times E(B-V)_{\mathrm{g}}$, following Calzetti et al. (1994). d) Same as c) for a $50 \%$ burst model.

Out of the several burst strengths we tried, we show the results for a moderate $(b=25 \%)$ and a strong burst model: $b=50 \%$, the maximum strength achievable without exhausting the gas reservoir. These models represent the simplest case, in which the presently observed burst of star-formation is the first interaction-triggered star formation event in these galaxies. We discuss in Sect. 4 the possibility of multiple starbursts.

The evolution of a few color indices is shown in Fig. 2 for the two burst strengths considered here. Color indices during the burst phase are to be compared with observed galaxy colors given in Table 1. We also show an example of a color index loop in the color-color diagram during the burst phase. The beginning of the burst and subsequent 140 Myr timesteps were marked along the color loop for burst ages in the range $40 \mathrm{Myr}-1$ Gyr. For comparison, observed colors of G1 and G4 are shown after the application of a standard inclination correction (these corrections are reported in Paper II and are supposed to account for the internal extinction expected for a given morphological type), and after a correction for internal extinction as determined from observed spectra, following Calzetti et al. (1994) as explained below. When the effects of internal extinction are taken into account, the strong burst model appears more suitable for explaining the observations. By also considering other color indices, we found that observed colors appear consistent with a burst age in the range $\sim 8-320 \mathrm{Myr}$. However, errors in color determination are quite large, as is visible from the error bars in Fig. 2, and corrections for internal extinction of total galaxy magnitudes/colors are very uncertain. The use of spectral features is particularly useful for improving this initial rough estimate of the burst age. Therefore, synthetic spectra calculated at intervals of $140 \mathrm{Myr}$ were compared with observed spectra within the age interval of interest.

Before comparison, a correction for internal extinction had to be applied to the observed spectra. As shown by Calzetti et al. (1994) and Calzetti (2001), the UV and optical continuum of starburst galaxies suffers from lower extinction than do the emission lines; in particular, the optical depth of the continuum underlying the Balmer lines is found to be about one-half of the difference between the optical depths of the Balmer emission lines. Therefore, as an initial estimate of the reddening affecting the optical continuum, we assumed $E(B-V)=0.5 \times E(B-$ $V)_{\mathrm{g}}$. We then varied $E(B-V)$ at steps of $0.05 \mathrm{mag}$ (or smaller) around this value. We applied this range of possible corrections by means of the IRAF task DEREDDEN assuming a Cardelli et al. (1989) extinction law with selective extinction $R_{V}=3.1$. 

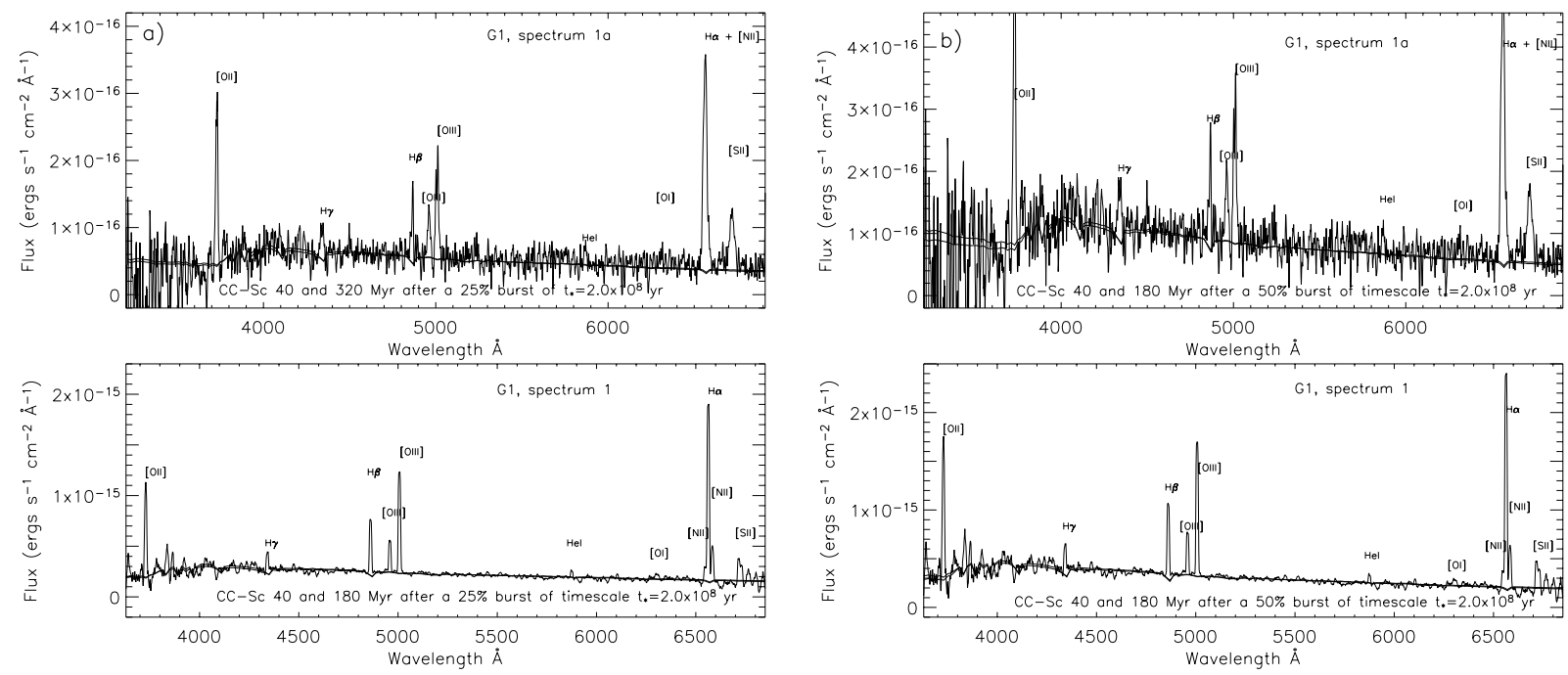

Fig. 3. a) CC-Sc model spectra calculated 40 (thick line) and 180 or 320 (thin lines) Myr after a $25 \%$ burst compared with the two spectra of $\mathrm{G} 1$ at $\mathrm{PA} 90^{\circ}($ top $)$ and $130^{\circ}$ (bottom) after internal reddening correction (see text). The reduced- $\chi^{2}$ is in the range $0.12-0.16$ (however, see discussion in Sect. 3). b) Same as a) for a model with a $50 \%$ burst. Model spectra at both burst ages acceptably reproduce the observed spectra. A minimum reduced $\chi^{2}=0.12$ is obtained for both spectra at age $180 \mathrm{Myr}$, while the $40 \mathrm{Myr}$ model gives a reduced $\chi^{2}=0.16$ and 0.15 for spectra 1a and 1 , respectively.

The resulting set of corrected spectra, normalized to their continuum flux around $5000 \AA$, was compared with normalized model spectra by means of an automated procedure based on $\chi^{2}$ evaluation. The average flux within several (10 to 12) intervals of continuum and the equivalent widths (EW) of $15 \mathrm{ab}-$ sorption lines ${ }^{4}$ were used for the calculation of the reduced- $\chi^{2}$. For each age in the interval of interest, the relevant model spectrum was compared to the whole set of observed spectra corrected for differing values of internal extinction, and the one giving the minimum reduced- $\chi^{2}$ was chosen. This way, each age had an associated $\chi^{2}$ and optimal value of $E(B-V)$. The age with minimum reduced- $\chi^{2}$ value was chosen as the best representation of the observed spectrum. In some cases the variation of reduced- $\chi^{2}$ between models at adjacent burst age values can be very small. In these circumstances, any of these ages could be reliable, not only the one with minimum reduced- $\chi^{2}$.

The final best values of $E(B-V)$ in case of $b=50 \%$ models were found to be $0.12 \mathrm{mag}$ and $0.30-0.35 \mathrm{mag}$ for spectra $1\left(\mathrm{PA}=130^{\circ}\right)$ and $1 \mathrm{a}\left(\mathrm{PA}=90^{\circ}\right.$, sampling a different portion of the galaxy, see Paper II) of G1, respectively. For the two available spectra of $\mathrm{G} 4$ (spectra 4 and $4+$ at $\mathrm{PA}=130^{\circ}$ and $90^{\circ}$ ) we found $E(B-V)=0.35$ and $0.49 \mathrm{mag}$, in agreement with the expectations from the measured $E(B-V)_{\mathrm{g}}$, taking the errors into account. Comparably good fits could be obtained with lower $E(B-V)$ values and $b=25 \%$ models. While these lower extinction values, $E(B-V)=0.23$ and $0.39 \mathrm{mag}$ for the two spectra, could still be acceptable within errors for G4, the best fit to the moderate burst model in the case of G1, especially for spectrum 1, would require far too low an extinction value, about $1 / 6$ of the measured $E(B-V)_{\mathrm{g}}$. This is a further indication that the moderate burst is not an adequate model for G1.

\footnotetext{
${ }^{4}$ In G1's spectra, absorption lines are very weak and, in most cases, hidden by emission lines, so only continuum intervals were considered for this galaxy.
}

In Figs. 3 to 5, we compare the extinction corrected spectra of G1 and G4 with model spectra for the two assumed burst strengths, conveniently scaled in intensity. Since the spectral resolution of the models $(20 \AA)$ is lower than that of observed spectra, a box-car smoothing was applied to the latter.

Spectrum 1, as presented in Paper II, was contaminated by an M-type star overlapped to G1 in projection on the sky. Therefore, in collapsing spectrum 1 of G1 along the spatial direction, we excluded those few columns contaminated by the star, which would compromise the comparison with model spectra. For this reason, the total intensity and continuum shape (in the red) of the spectrum presented in Fig. 3 differ from those shown in Paper II.

The models displayed in Fig. 3a are sufficiently good approximations of the observed spectra, with reduced- $\chi^{2}$ in the range 0.12-0.16; however, best-fits were obtained for exceedingly low values of internal extinction with respect to initial estimates based on the Balmer decrement, as noted above. In the case of both moderate and strong bursts (Figs. 3a and b), the models that approach the observed spectra of G1 better are those at a burst age in the range 40-180 Myr, with the oldest age favored by the reduced- $\chi^{2}$ we calculated.

For G4, we show the comparison with spectra 4 and 4+ in Figs. 4 and 5. In these spectra, the most important absorption lines are detected and the Ca II absorption index (Rose 1984; Leonardi \& Rose 1996) is typical of A0- or B-type stars. For the moderate-burst models (Fig. 4), best-fits were obtained after correcting for an internal extinction in the range $E(B-V)=$ 0.39 (younger ages) -0.44 mag (older age). The $40 \mathrm{Myr}$ model is the one that approaches the observations better, taking into account an internal extinction $E(B-V)=0.39 \mathrm{mag}$. For the strong-burst models (Fig. 5), best-fits were obtained by considering an internal extinction $E(B-V)=0.49$ mag for sepctrum $4+$, and in the range $0.33-0.35$ mag for spectrum 4 . Also 

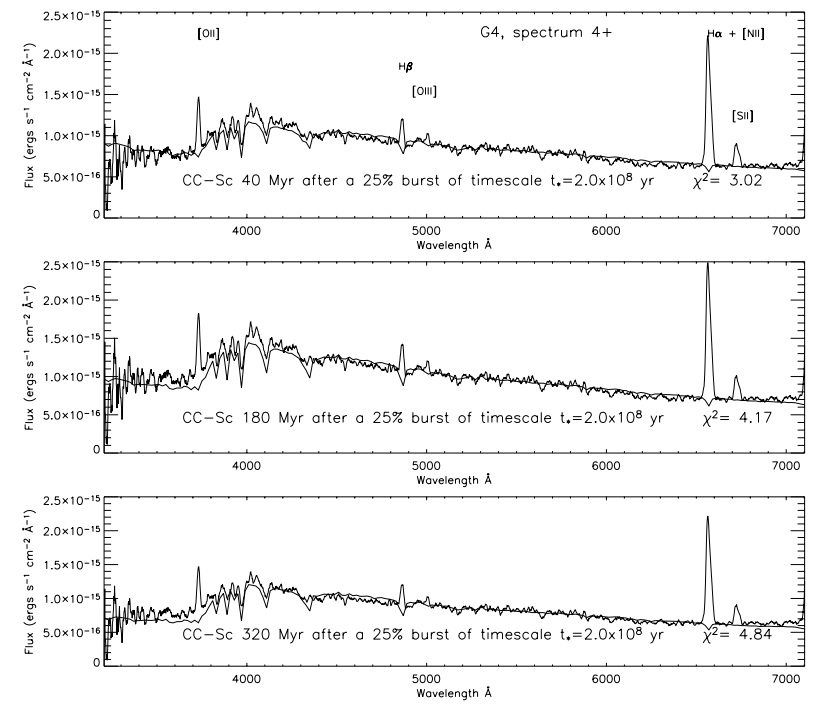

Fig. 4. CC-Sc model spectra calculated 40, 180, and 320 Myr (from top to bottom, thick line) after a $25 \%$ burst compared with the spectrum of $\mathrm{G} 4\left(\right.$ at $\left.\mathrm{PA}=90^{\circ}\right)$ after internal reddening correction (see text). The reduced- $\chi^{2}$ of the fit for the 3 model ages based on continuum intervals and absorption line equivalent widths, minimized over a range of possible internal extinction values, is indicated.

in this case, model spectra calculated 40 and $180 \mathrm{Myr}$ after the strong burst are those that approach better the observed spectra, the youngest age being favored by the reduced- $\chi^{2}$. The model spectra at burst ages of 320 and $740 \mathrm{Myr}$ are also displayed to show how models with older burst ages $(\geq 320 \mathrm{Myr})$ depart from the observations.

The optical-NIR spectral energy distribution (SED) served for further comparison between the models and observational data. The SED diagrams (see, e.g., Krüger et al. 1995) were obtained from integrated broad-band fluxes convolved with the Johnson filter response functions (Bessell 1990) and normalized to the flux in the $V(\mathrm{G} 1)$ or $R(\mathrm{G} 4)$ band, as a function of the effective wavelength $\lambda_{\text {eff }}$ of the filters. Flux values for the models, in units of erg $\mathrm{cm}^{-2} \mathrm{~s}^{-1}$, were obtained from the absolute magnitudes calculated from the model spectra by a GALEV sub-routine. Flux values for the galaxies were obtained from the measured magnitudes and, in the optical regime, also from their spectra. The available spectral ranges allowed us to derive $B, V$ fluxes for $\mathrm{G} 1$ and $B, V, R$ fluxes for G4. Photometric zero points, as determined from the observed spectrophotometric standard stars, were included. The diagrams are shown in Figs. 6, 7 for the two CC-Sc models at several burst ages. For G1, SEDs obtained from the strong burst model offer better agreement with the data, while for G4 both burst strengths produce SEDs in good agreement with the observations. Models with a burst age in the range 40 to $180 \mathrm{Myr}$ are favored, thus confirming the results from the spectral analysis above.

We note that for both galaxies the normalized spectrophotometric optical fluxes are slightly higher than the photometric values in the same bands ( $B$ band for $\mathrm{G} 1$ and both $B$ and $V$ bands for G4) and are in very good agreement with the modeled SEDs (for strong burst). We interpret this fact as a consequence of insufficient correction of the observed magnitudes for internal reddening. While spectra were corrected for internal reddening following the procedure described above, only a standard inclination correction was applied to the total magnitudes (Paper II). The reason for this choice is that we are not able to determine the amount of internal extinction affecting the total magnitudes of the galaxies with sufficient accuracy. The dust distribution in G1 is inhomogeneous, as shown by the differing color excesses measured in spectra across distinct portions of the galaxy $\left(E(B-V)_{\mathrm{g}}=0.17\right.$, 0.35, 0.65 mag; see Paper II). A similar situation holds for G4 (see Paper IV), as already mentioned. Hence, it is not clear which value of extinction correction is more suitable for application to total magnitudes. By using as reference the average color excess measured from different spectra, $E(B-V)_{\mathrm{g}}=$ 0.5 mag for G1 and 0.6 mag for G4, we show in Fig. 2 how the galaxies would be placed on a two-color diagram after correction for internal extinction, under the assumption that the stellar continuum is affected by roughly half of the extinction affecting the emission lines (Calzetti et al. 1994). This is to be considered only an indication of the effects of internal extinction on galaxy colors, so we prefer not to use magnitudes and colors corrected this way in the final comparison between observed and modeled quantities. At least for G4, there is an observational indication (Paper IV, Fig. 7) that an amount of extinction that is significantly higher than the one assumed by the standard inclination correction is present across the galaxy. This is also probably the case for G1. Since shorter wavelengths are more affected by extinction, the effects of an insufficient correction are expected to be greater in $B$ than in $V$, as actually appears from the comparison between photometric and spectrophotometric points in Fig. 7.

The normalized NIR observational fluxes of G1 appear higher than the modeled ones (i.e. shifted toward older ages), but still in agreement within the errors ${ }^{5}$. This discrepancy in the NIR is most probably a consequence of contamination from the foreground star projected onto G1 (see Fig. 1), which displays a typical M-type spectrum and could not be separated from the galaxy itself when doing NIR photometry.

Observational and modeled colors and metallicities are shown in Table 1 for CC-Sc models with different burst strengths and ages. In Table 2 absolute magnitudes of G1 and G4 (distance modulus $m-M=36.276$ ) are compared with model ones, calculated for burst strengths $b=0.5$ and $b=0.25$. Model luminosities, masses, and SFRs were rescaled taking as reference the $R$-band luminosity of $\mathrm{G} 1$ and the $K$-band luminosity of G4. Once again we stress that a standard inclination correction was applied to observed colors and magnitudes to account for internal extinction, but this correction was probably insufficient, as suggested by the Balmer decrement measured in the individual spectra. In fact, the discrepancies between

\footnotetext{
5 The error bars in Fig. 6 represent only the error in the observed magnitudes (see Paper II for a discussion of photometric errors). Comparable errors have to be considered for the model SEDs. The errors of modeled colors are estimated as $0.05 \mathrm{mag}$ in the optical and $0.1 \mathrm{mag}$ in the NIR due to uncertainties in the isochrones and color calibrations.
} 

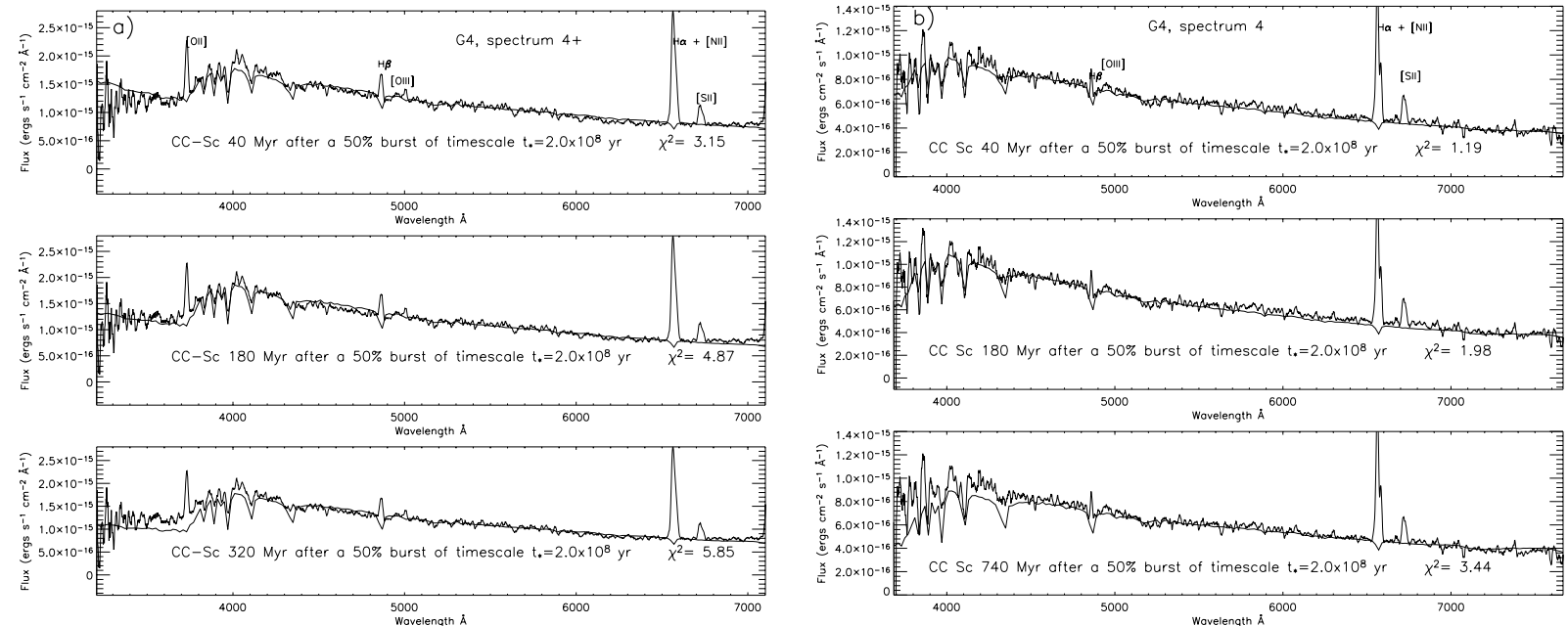

Fig. 5. a) CC-Sc model spectra calculated 40,180, and $320 \mathrm{Myr}$ (from top to bottom, thick line) after a 50\% burst compared with the spectrum of G4 at PA $=90^{\circ}$, after internal reddening correction (see text). Reduced- $\chi^{2}$ values are indicated. The first two models are in good agreement with the data, while the third one departs from the observations in the blue part of the spectrum. b) Same model as in a) compared to the spectrum of $\mathrm{G} 4$ at $\mathrm{PA}=130^{\circ}$ for burst ages 40,180 , and 740 Myr. Reduced- $\chi^{2}$ values are marked. The older burst model departs from the observations significantly, while the first two models match the data reasonably well.
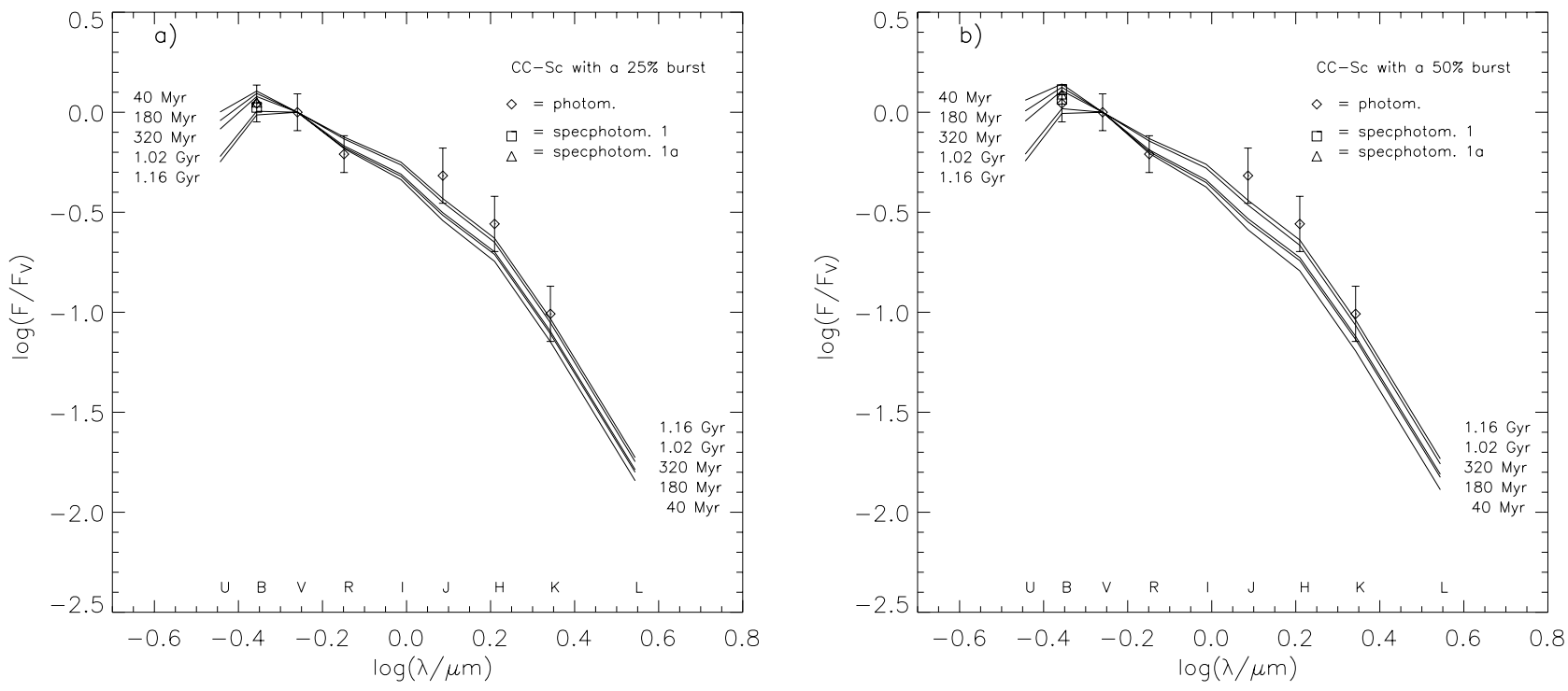

Fig. 6. Energy distribution normalized to the $V$ band for CC-Sc models in a wide range of burst ages after the onset of a $25 \%$ [a)] and a $50 \%$ [b)] burst. Diamonds with associated error bars are photometric observational points for G1, after correction for inclination. The square and the triangle are spectrophotometric points obtained from the observed, dereddened, spectra 1 and 1a after convolution with $B$ and $V$ filter response functions (Bessell 1990).

photometric measurements in the $B$ and $V$ bands and the model SEDs visible in Figs. 6, 7 are found also in the direct comparison between observed and modeled colors and luminosities. Taking this fact into account, the comparison between observed and model luminosities indicates that G1 is roughly consistent with a total mass (star + gas) in the range $4-6 \times 10^{9} M_{\odot}$ (using as reference the strong burst models) and G4 has luminosities in good agreement with $\sim 2-3 \times 10^{10} M_{\odot}$ models 40 to $180 \mathrm{Myr}$ after a moderate to strong burst. The modeled stellar mass-tolight ratios in the $K$ band are $0.62(0.65)$ and $0.46(0.55) M_{\odot} L_{\odot}^{-1}$ for a 40 and a 180 Myr old strong (moderate) burst, respectively. The metallicities obtained with chemically consistent models are comparable to (or slightly higher than) those determined from the observed emission-line ratios. The SFRs obtained from models with burst age 40 Myr (taking the assumed FVM $=0.5$ into account, see Table 2) are in reasonable agreement with measurements for G1. In the case of G4, models with SFRs in better agreement with the observations are those with burst age $180 \mathrm{Myr}$, while models with a younger age give too high a SFR. As already mentioned in Sect. 2.1 and in Paper IV, the internal extinction map of G4 strongly suggests that the SFR derived from the $\mathrm{H} \alpha$ emission-line is a lower limit to the actual SFR, and continuum radio observations suggest that the actual SFR of G4 is $\gtrsim 6 M_{\odot} \mathrm{yr}^{-1}$, i.e. close to the SFR for the strong burst model with age $180 \mathrm{Myr}$. 

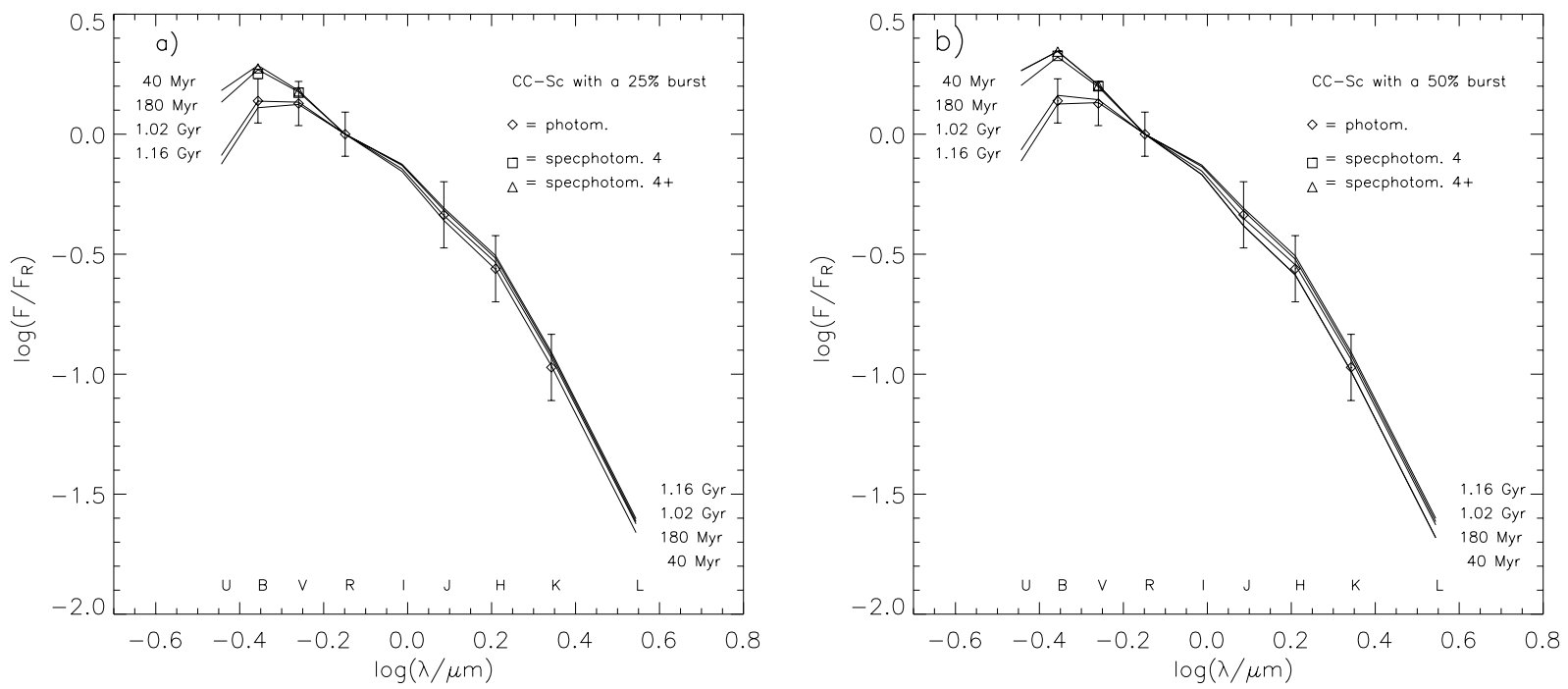

Fig. 7. SEDs for the CC-Sc models at differing ages after a weak [a)] and a strong [b)] burst. Photometric points of G4 are marked with diamonds, while the spectrophotometric points derived from the dereddened spectra (according to the best-fit extinction values obtained from the spectral analysis) at PA $130^{\circ}$ and $90^{\circ}$ are indicated by triangles and squares, respectively. For models of both strengths, optical spectrophotometric points and NIR photometric points agree very well with a burst age in the range 40 to $180 \mathrm{Myr}$. The thick line indicates the best-fit age obtained from the spectra.

Table 2. G1 and G4: observed ${ }^{a}$ and modeled luminosities.

\begin{tabular}{llllllll}
\hline \hline Object/Model & $M_{B}$ & $M_{V}$ & $M_{R}$ & $M_{J}$ & $M_{H}$ & $M_{K}$ & $S F R\left(M_{\odot} \mathrm{yr}^{-1}\right)$ \\
\hline G1 & -18.92 & -19.39 & -19.70 & -21.30 & -21.73 & -21.82 & 2.8 \\
& & & & & & & \\
CC-Sc, $b=0.5$ & & & & & & & \\
$40 \mathrm{Myr}, M=5.96 \times 10^{9} M_{\odot}$ & -19.09 & -19.35 & -19.70 & -20.58 & -21.07 & -21.26 & 4.3 \\
$180 \mathrm{Myr}, M=4.12 \times 10^{9} M_{\odot}$ & -19.15 & -19.38 & -19.70 & -20.62 & -21.14 & -21.35 & 1.5 \\
$320 \mathrm{Myr}, M=3.92 \times 10^{9} M_{\odot}$ & -19.10 & -19.37 & -19.70 & -20.69 & -21.25 & -21.48 & 0.69 \\
& & & & & & & \\
CC-Sc, $b=0.25$ & & & & & & & \\
$40 \mathrm{Myr}, M=7.23 \times 10^{9} M_{\odot}$ & -18.92 & -19.29 & -19.70 & -20.66 & -21.17 & -21.37 & 2.6 \\
$180 \mathrm{Myr}, M=5.74 \times 10^{9} M_{\odot}$ & -19.01 & -19.32 & -19.70 & -20.67 & -21.20 & -21.40 & 1.0 \\
$320 \mathrm{Myr}, M=5.57 \times 10^{9} M_{\odot}$ & -18.97 & -19.31 & -19.70 & -20.72 & -21.27 & -21.49 & 0.49 \\
& & & & & & & \\
G4 & -20.30 & -20.86 & -21.36 & -22.40 & -22.87 & -23.06 & 3.2 \\
& & & & & & & \\
CC-Sc, $b=0.5$ & & & & & & & \\
$40 \mathrm{Myr}, M=3.11 \times 10^{10} M_{\odot}$ & -20.88 & -21.15 & -21.49 & -22.38 & -22.87 & -23.06 & 22.1 \\
$180 \mathrm{Myr}, M=1.98 \times 10^{10} M_{\odot}$ & -20.88 & -21.09 & -21.40 & -22.32 & -22.85 & -23.06 & 7.0 \\
$320 \mathrm{Myr}, M=1.68 \times 10^{10} M_{\odot}$ & -20.68 & -20.94 & -21.28 & -22.27 & -22.83 & -23.06 & 2.9 \\
CC-Sc, $b=0.25$ & & & & & & & \\
$40 \mathrm{Myr}, M=3.44 \times 10^{10} M_{\odot}$ & -20.61 & -20.98 & -21.39 & -22.35 & -22.86 & -23.06 & 12.2 \\
$180 \mathrm{Myr}, M=2.64 \times 10^{10} M_{\odot}$ & -20.66 & -20.97 & -21.35 & -22.32 & -22.85 & -23.06 & 4.6 \\
$320 \mathrm{Myr}, M=2.38 \times 10^{10} M_{\odot}$ & -20.54 & -20.89 & -21.27 & -22.30 & -22.85 & -23.06 & 2.1 \\
\hline
\end{tabular}

${ }^{a}$ Observed luminosities after the application of a standard inclination correction.

In the strong burst model, the mass percentage of stars produced since the onset of the burst is $\approx 9 \%$ after $40 \mathrm{Myr}$ and $24 \%$ after $180 \mathrm{Myr}$, and they contribute $44 \%$ and $63 \%$ of the visual light.

\section{Early-type group member: G2}

Both photometric and spectroscopic results (Paper II) have shown that G2 is a strongly reddened early-type galaxy with evidence of present-day star-formation activity at its center. 
The Balmer decrement in its spectrum implies an internal extinction $A_{\mathrm{V}}=1.6 \mathrm{mag}$. Although such a high extinction value is probably due mostly to dust associated with the central starformation, it is possible that the inclination correction we applied to the total magnitudes $\left(A_{\mathrm{V}}=0.8\right.$, see Paper II) was insufficient to account for the amount of extinction affecting this galaxy, as in the case of the two spiral galaxies discussed above. Nevertheless, we preferred to maintain the standard inclination correction concerning total magnitudes, because it is unclear whether the extinction value derived from the long-slit spectrum can be extended to the whole galaxy. And, due to the low signal-to-noise ratio of the $\mathrm{H} \beta$ emission line, the uncertainty in the derived extinction is quite high $( \pm 1.2 \mathrm{mag})$.

The strong Balmer absorption lines (in which the emission lines are embedded) and the CaII absorption index suggest that the spectrum is dominated by a population of A-type stars. The emission line ratios are consistent with a subsolar metallicity of the gaseous component $\left(Z \lesssim 0.5 Z_{\odot}\right.$ ).

In Paper IV (Sect. 7.1), we proposed two possible explanations for the observed properties of G2: either this galaxy is the outcome of a merger that induced a (now fading) burst of star formation and produced part of the faint tails and plumes visible in the group, or it is an early-type galaxy that started a minor episode of star-formation as a consequence of tidal interaction with the companion spiral galaxies. In fact, several numerical simulations have shown that interactions and merger events may trigger gas inflows and subsequent central star-formation (e.g. Noguchi 1988; Barnes \& Hernquist 1991; Mihos et al. 1993; Mihos \& Hernquist 1994; Barnes \& Hernquist 1996). Here we used evolutionary synthesis models to investigate both possibilities. To test the first possibility, we simulated a burst of star-formation induced by the merger of two early-type spiral galaxies, i.e. two $\mathrm{Sa}$ or $\mathrm{Sb}$ galaxies (at the moment GALEV does not support the simulation of mixedmorphology mergers). We investigated the second possibility using the model of an elliptical (E) galaxy in which we turn on a burst of star-formation at late ages.

\subsection{Early type spiral-spiral merger}

We compared the observational data with two models in which the progenitors are two $\mathrm{Sa}$ or Sb galaxies. A burst of starformation with exponential decay time-scale $t_{*}=2.0 \times 10^{8} \mathrm{yr}$ was turned on when the galaxies were $11 \mathrm{Gyr}$ old $^{6}$. The color evolution for these two models and an example of color loop in a color-color diagram during the burst phase are shown in Fig. 8. The position of G2 in the color-color diagrams is shown after a standard inclination correction (empty diamond) and as it would appear after a correction for internal extinction using the color excess derived from the Balmer decrement, $E(B-V)_{\mathrm{g}}=0.52 \mathrm{mag}$ (but see the above remarks concerning the application of the extinction correction to total magnitudes). Photometric data appear consistent with an already aging burst (age $>0.5 \mathrm{Gyr}$ ), as do the A-star spectral features.

\footnotetext{
${ }^{6}$ Turning on the burst in a later epoch (e.g. 12 Gyr) would not change the results significantly.
}
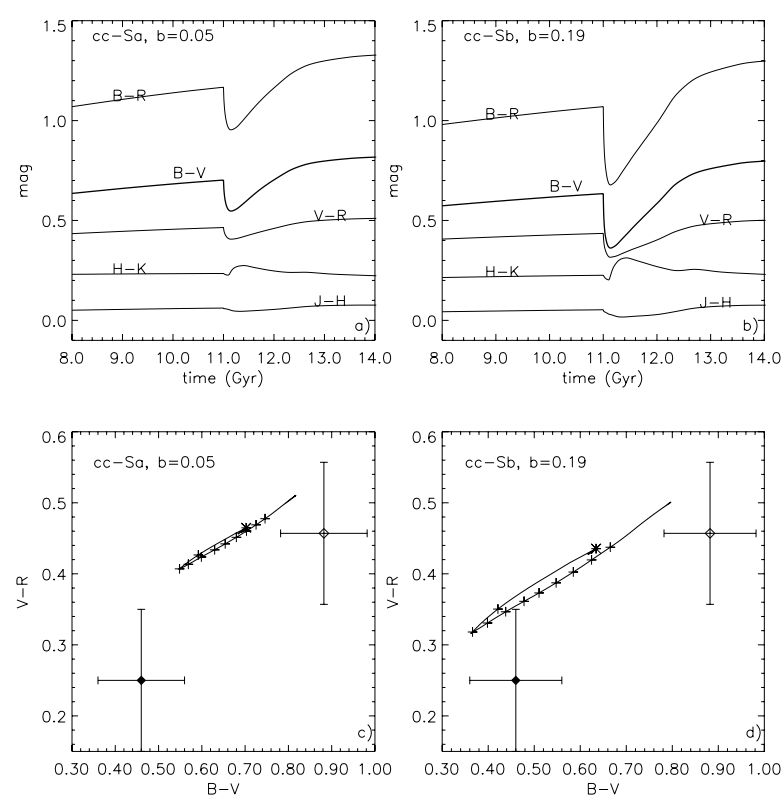

Fig. 8. a) Evolution of color indices for a cc-Sa model with a $5 \%$ burst switched on after $11 \mathrm{Gyr}$ of undisturbed evolution. The $B-V$ index is indicated with a thick line to avoid confusion with other indices. For the same reason, an offset of -0.5 was applied to the $J-H$ index. b) Same as a), for cc-Sb model with a $19 \%$ burst. c) Color-color diagram showing the color indices evolution during the burst phase for a cc-Sa model with a 5\% burst. The asterisk marks the onset of the burst at a galaxy age of $11 \mathrm{Gyr}$. Plus symbols are plotted for burst ages from $40 \mathrm{Myr}$ to $1.28 \mathrm{Gyr}$ at intervals of $140 \mathrm{Myr}$. The empty diamond indicates the observed colors of G2, after a standard inclination correction. The full diamond indicates the observed colors after correction for internal extinction using $E(B-V)_{\mathrm{g}}=0.52 \mathrm{mag}$, as derived from the Balmer decrement. d) Same as c) for a cc-Sb model with a $19 \%$ burst.

In the Sa model only a weak burst, with maximum strength $\sim 5 \%$, can be simulated due to the limited amount of gas remaining in the galaxy after $11 \mathrm{Gyr}$ of undisturbed evolution. According to the CaII absorption index, the model spectra $<0.5$ Gyr after the burst show a stellar population that is too young with respect to the observations. Unlike the case of the two spiral galaxies, we did not take $0.5 \times E(B-V)_{\mathrm{g}}$ as the initial guess for the internal extinction in the continuum, $E(B-V)$, because this relation was derived by Calzetti et al. (1994) for starburst galaxies, which G2 is not (anymore). However, since not only the observed spectrum but also relative photometry suggested a strong reddening, we used the value determined from the Balmer decrement as an upper limit to $E(B-V)$ and applied an analogous fitting procedure to the one described in Sect. 3 to determine the optimal extinction value, together with the best fit burst age. The value of $E(B-V)$ derived this way is $\approx 0.4 \mathrm{mag}$ (specifically in the range $0.36-0.41 \mathrm{mag}$ ), which is somewhat lower than the measured $E(B-V)_{\mathrm{g}}$, although well within the error of the measurement. The best agreement with the extinction-corrected, smoothed spectrum is found $900 \mathrm{Myr}$ after the onset of the burst (minimum reduced- $\chi^{2}=7.34$ ); however, also model spectra at burst ages 620 and $760 \mathrm{Myr}$ give nearly as good a representation of the observed spectrum (reduced $-\chi^{2}=7.38$ and 7.36, respectively; Fig. 9). We note 

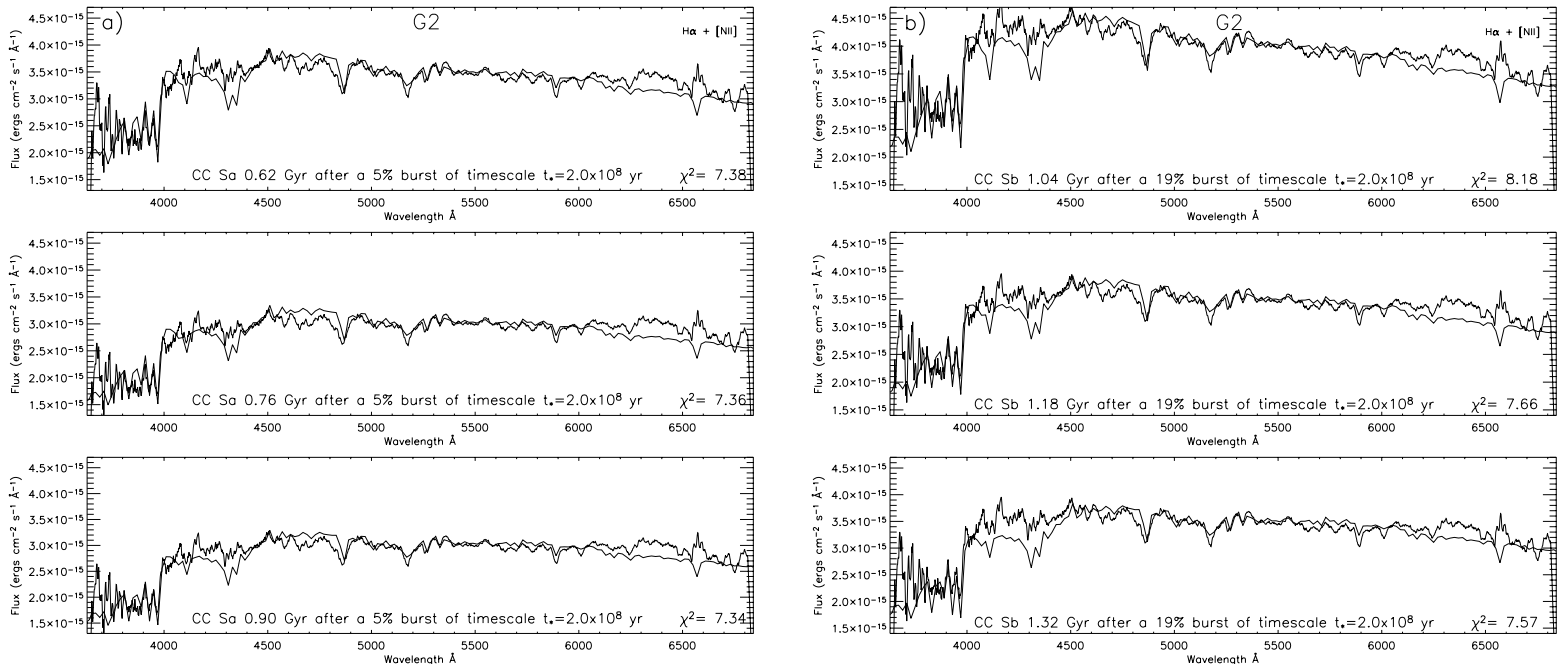

Fig. 9. a) CC-Sa model spectra (thick line), conveniently scaled, overplotted to the dereddened and smoothed observed spectrum of G2 at three different ages after the onset of a 5\% burst. The minimum reduced $\chi^{2}$ is obtained for $E(B-V)=0.36$ mag and burst age 900 Myr; however, the other two models shown give nearly as good a result with $E(B-V)=0.41$ and 0.36 mag at ages 620 and $760 \mathrm{Myr}$, respectively. The fit worsens noticeably at younger/older ages than those displayed. b) Same as a) for a CC-Sb model at three different ages after the onset of a 19\% burst. The best fit occurs for $E(B-V)=0.41 \mathrm{mag}$ and burst ages between 1.2 and $1.3 \mathrm{Gyr}$.

Table 3. G2: observed ${ }^{a}$ and modeled colors.

\begin{tabular}{lllllllll}
\hline \hline Object/Model & $B-V$ & $V-R$ & $V-K$ & $B-R$ & $B-H$ & $J-H$ & $H-K$ & $Z / Z_{\odot}$ \\
\hline G2 & 0.88 & 0.46 & 2.52 & 1.34 & 3.24 & 0.59 & 0.16 & $\sim 0.5$ \\
CC-Sa, $M=2.5 \times 10^{10} M_{\odot}, b=0.05$ & & & & & & & & \\
$480 \mathrm{Myr}$ & 0.59 & 0.52 & 2.48 & 1.11 & 2.85 & 0.57 & 0.22 & 0.68 \\
$620 \mathrm{Myr}$ & 0.62 & 0.54 & 2.49 & 1.15 & 2.89 & 0.57 & 0.22 & 0.67 \\
$760 \mathrm{Myr}$ & 0.65 & 0.55 & 2.50 & 1.19 & 2.93 & 0.57 & 0.22 & 0.64 \\
$900 \mathrm{Myr}$ & 0.67 & 0.56 & 2.51 & 1.23 & 2.97 & 0.56 & 0.22 & 0.60 \\
$\mathrm{CC}-\mathrm{Sb}, M=2.5 \times 10^{10} M_{\odot}, b=0.19$ & & & & & & & & \\
$900 \mathrm{Myr}$ & 0.55 & 0.49 & 2.36 & 1.04 & 2.70 & 0.55 & 0.22 & 0.64 \\
$1.04 \mathrm{Gyr}$ & 0.59 & 0.50 & 2.39 & 1.09 & 2.76 & 0.55 & 0.22 & 0.61 \\
$1.18 \mathrm{Gyr}$ & 0.63 & 0.52 & 2.42 & 1.15 & 2.83 & 0.55 & 0.21 & 0.59 \\
$1.32 \mathrm{Gyr}$ & 0.67 & 0.54 & 2.45 & 1.21 & 2.91 & 0.55 & 0.21 & 0.57 \\
\hline
\end{tabular}

${ }^{a}$ Observed colors after the application of a standard inclination correction.

that the $\mathrm{H} \beta$ absorption line becomes weaker at ages $\gtrsim 760 \mathrm{Myr}$, while it matches the observed feature better at a younger burst age.

In the $\mathrm{Sb}$ model, at the same galaxy age, the remaining gas content is higher, so a stronger burst can be turned on. We considered a $19 \%$ burst turned on at a galaxy age of 11 Gyr. In this case the signatures of A-type stellar populations remain visible for a longer time after the start of the burst and begin fading only after $\gtrsim 1.3 \mathrm{Gyr}$, when the Balmer absorption lines weaken and the CaII absorption index increases toward unity. The best agreement with the observed spectrum is found to be $\sim 1.2$ to 1.3 Gyr after the onset of the burst (Fig. 9).

Both models, obtained considering a chemically consistent evolution, provide a satisfactory match to the metallicity-

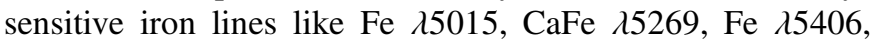
Fe $\lambda 5709$, and Fe $\lambda 5782$ (Worthey 1994). The gas metallicity reached by the $\mathrm{Sb}$ model at the best-fit age is $Z=0.0106 \sim$ $0.59 Z_{\odot}$, in good agreement with the metallicity derived in Paper II from the emission-line ratios, whereas it is slightly higher for the Sa model (Table 3).

In Table 3 the observed, face-on colors are compared with colors from the two models at four different burst ages each. The color comparison favors the older burst models, in particular the Sa model at burst ages of 760-900 Myr. Significant discrepancies are found in the colors involving the $B$ band. In fact in all models, these are bluer than observed. In connection with this fact, we note that on the SED diagrams in Fig. 10 the spectrophotometric flux in the $B$ band is higher than the photometric one, as in the case of G1 and G4. The normalized $B$ band spectrophotometric flux and the photometric fluxes in the other bands appear to largely agree with the model SEDs. Once again, the Sa model is the one that approaches better 
Table 4. G2: observed ${ }^{\mathrm{a}}$ and modeled luminosities.

\begin{tabular}{llllllll}
\hline \hline Object/Model & $M_{B}$ & $M_{V}$ & $M_{R}$ & $M_{J}$ & $M_{H}$ & $M_{K}$ & $S F R\left(M_{\odot} \mathrm{yr}^{-1}\right)$ \\
\hline G2 & -19.82 & -20.70 & -21.16 & -22.47 & -23.06 & -23.22 & $0.4-0.8$ \\
CC-Sa, $b=0.05$ & & & & & & & \\
$480 \mathrm{Myr}, M=3.19 \times 10^{10} M_{\odot}$ & -20.15 & -20.74 & -21.27 & -22.43 & -23.00 & -23.22 & 0.44 \\
$620 \mathrm{Myr}, M=3.28 \times 10^{10} M_{\odot}$ & -20.11 & -20.73 & -21.26 & -22.43 & -23.00 & -23.22 & 0.22 \\
$760 \mathrm{Myr}, M=3.37 \times 10^{10} M_{\odot}$ & -20.07 & -20.72 & -21.26 & -22.43 & -23.00 & -23.22 & 0.12 \\
$900 \mathrm{Myr}, M=3.45 \times 10^{10} M_{\odot}$ & -20.03 & -20.71 & -21.26 & -22.44 & -23.00 & -23.22 & 0.06 \\
$\mathrm{CC}-\mathrm{Sb}, b=0.19$ & & & & & & & \\
$900 \mathrm{Myr}, M=2.72 \times 10^{10} M_{\odot}$ & -20.30 & -20.86 & -21.34 & -22.45 & -23.00 & -23.22 & 0.14 \\
$1.04 \mathrm{Gyr}, M=2.77 \times 10^{10} M_{\odot}$ & -20.24 & -20.83 & -21.34 & -22.46 & -23.00 & -23.22 & 0.07 \\
$1.18 \mathrm{Gyr}, M=2.75 \times 10^{10} M_{\odot}$ & -20.17 & -20.80 & -21.32 & -22.45 & -23.00 & -23.22 & 0.04 \\
$1.32 \mathrm{Gyr}, M=2.69 \times 10^{10} M_{\odot}$ & -20.10 & -20.77 & -21.31 & -22.45 & -23.00 & -23.22 & 0.02 \\
\hline
\end{tabular}

${ }^{a}$ Observed luminosities after the application of a standard inclination correction.
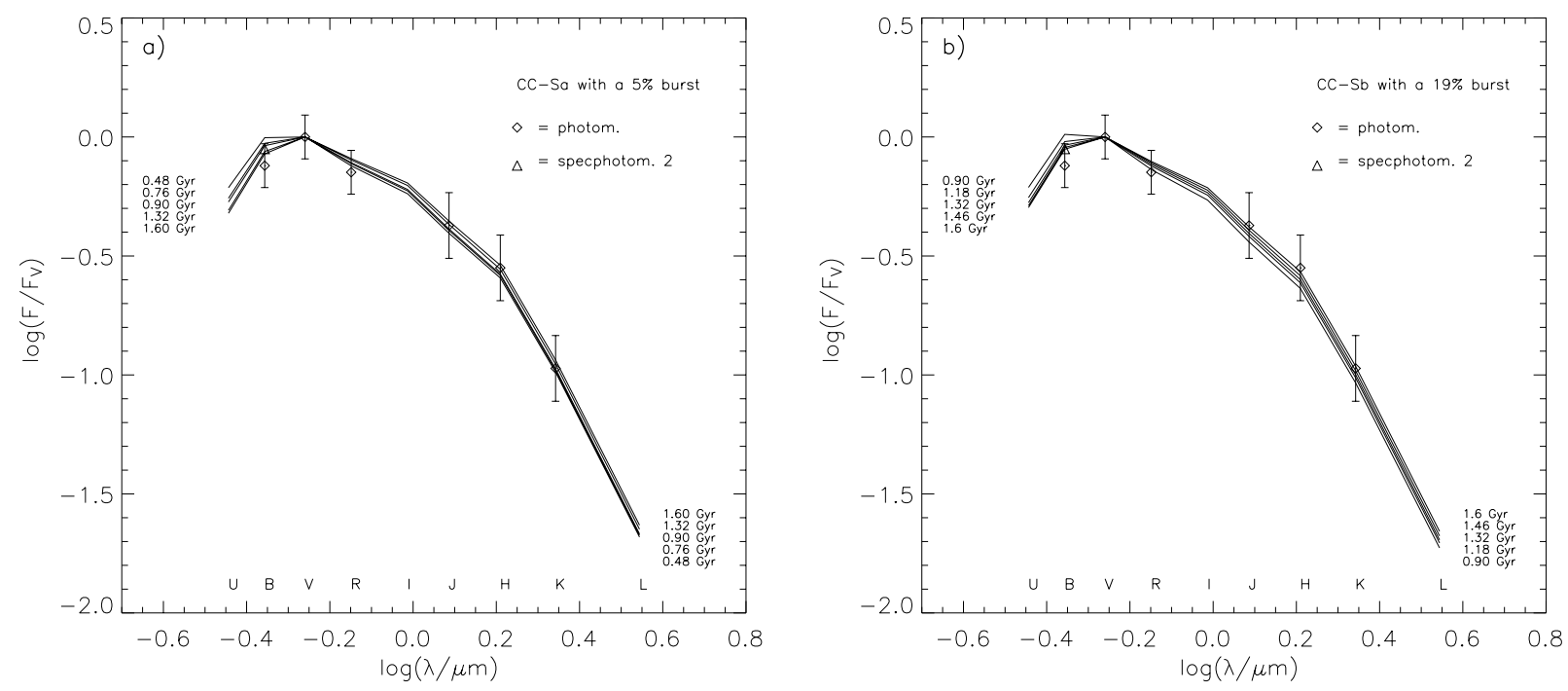

Fig. 10. SEDs calculated for a wide range of burst ages for the CC-Sa [a)] and Sb [b)] models, along with dereddened photometric and spectrophotometric data points for G2. The thick lines indicate the best-fit models according to the spectral analysis.

the observations. The discrepancy in the $B$ band would confirm an insufficient extinction correction of the observed magnitude that is particularly visible in $B$, where extinction effects are stronger.

Model luminosities and total (star + gas) masses, rescaled to the $K$-band observed luminosity, are listed in Table 4 , together with observed luminosities. Except for the $B$-band, where the measured luminosity is probably underestimated, the older burst ages reasonably agree with the observations, especially for the Sa model. The total mass of G2 implied by the Sa and $\mathrm{Sb}$ models would be $\sim 3.4 \times 10^{10}$ and $2.7 \times 10^{10} M_{\odot}$, respectively. This is equivalent to assuming an equal mass merger of two $\mathrm{Sa}(\mathrm{Sb})$ galaxies of mass $1.7 \times 10^{10}\left(1.4 \times 10^{10}\right) M_{\odot}$ each, which led to the observed S0 morphology. Predicted SFRs rescaled to the above total masses are given in Table 4. The agreement with observations - taking into account the errors in the observations and in the models - is reasonable for the $\mathrm{Sa}$ model, while SFRs predicted by the Sb model are too low compared to the measured values.
According to the $\mathrm{Sa}(\mathrm{Sb})$ model, the stellar mass of $\mathrm{G} 2$ is $3.4 \times 10^{10}\left(2.5 \times 10^{10}\right) M_{\odot}$, where $5 \%(11 \%)$ was produced in a $\sim 0.7-0.9(1.2-1.3)$ Gyr old burst. Stars produced during the burst contribute $\sim 6-3 \%(10-8 \%)$ of the visual light.

\subsection{Burst in an elliptical galaxy}

To test the possibility that G2 is an early-type, gas-poor galaxy that experienced an episode of star-formation as a consequence of an interaction with or gas accretion from the companion(s), we used a CC model with an E-type SFH and turned on an episode of star-formation at a galaxy age of $11 \mathrm{Gyr}$; the results would not change appreciably for an onset of the burst at later ages. At such a late age the gas reservoir of the E-type model-galaxy has already been replenished by evolved stars, so it is possible to simulate relatively strong bursts. We could not use an S0 model for this purpose, since the SFH assumed for S0 leaves the galaxy with a reservoir of gas that is too small at late ages, so that a sufficiently strong burst cannot be 
Table 5. G2 - Observed ${ }^{a}$ and $Z=0.008$ E model colors and luminosities.

\begin{tabular}{llllllll}
\hline \hline Object/Model & $B-V$ & $V-R$ & $V-K$ & $B-R$ & $B-H$ & $J-H$ & $H-K$ \\
& $M_{B}$ & $M_{V}$ & $M_{R}$ & $M_{J}$ & $M_{H}$ & $M_{K}$ & $S F R\left(M_{\odot} \mathrm{yr}^{-1}\right)$ \\
\hline $\mathrm{G} 2$ & 0.88 & 0.46 & 2.52 & 1.34 & 3.24 & 0.59 & 0.16 \\
& -19.82 & -20.70 & -21.16 & -22.47 & -23.06 & -23.22 & $0.4-0.8$ \\
$Z=0.008 \mathrm{E}, b=0.11,760 \mathrm{Myr}$ & 0.60 & 0.54 & 2.67 & 1.14 & 3.02 & 0.61 & 0.24 \\
$M=6.75 \times 10^{10} M_{\odot}$ & -19.96 & -20.55 & -21.09 & -22.37 & -22.98 & -23.22 & 0.20 \\
$Z=0.008 \mathrm{E}, b=0.11,900 \mathrm{Myr}$ & 0.64 & 0.56 & 2.68 & 1.20 & 3.09 & 0.61 & 0.24 \\
$M=6.94 \times 10^{10} M_{\odot}$ & -19.90 & -20.54 & -21.10 & -22.38 & -22.99 & -23.22 & 0.10 \\
$Z=0.008 \mathrm{E}, b=0.11,1.04 \mathrm{Gyr}$ & 0.68 & 0.57 & 2.70 & 1.25 & 3.14 & 0.61 & 0.24 \\
$M=7.03 \times 10^{10} M_{\odot}$ & -19.84 & -20.52 & -21.09 & -22.38 & -22.98 & -23.22 & 0.05 \\
$Z=0.008 \mathrm{E}, b=0.11,1.18 \mathrm{Gyr}$ & 0.72 & 0.59 & 2.72 & 1.30 & 3.20 & 0.61 & 0.23 \\
$M=7.03 \times 10^{10} M_{\odot}$ & -19.79 & -20.50 & -21.09 & -22.38 & -22.99 & -23.22 & 0.03 \\
\hline
\end{tabular}

${ }^{a}$ Observed luminosities and colors after the application of a standard inclination correction.
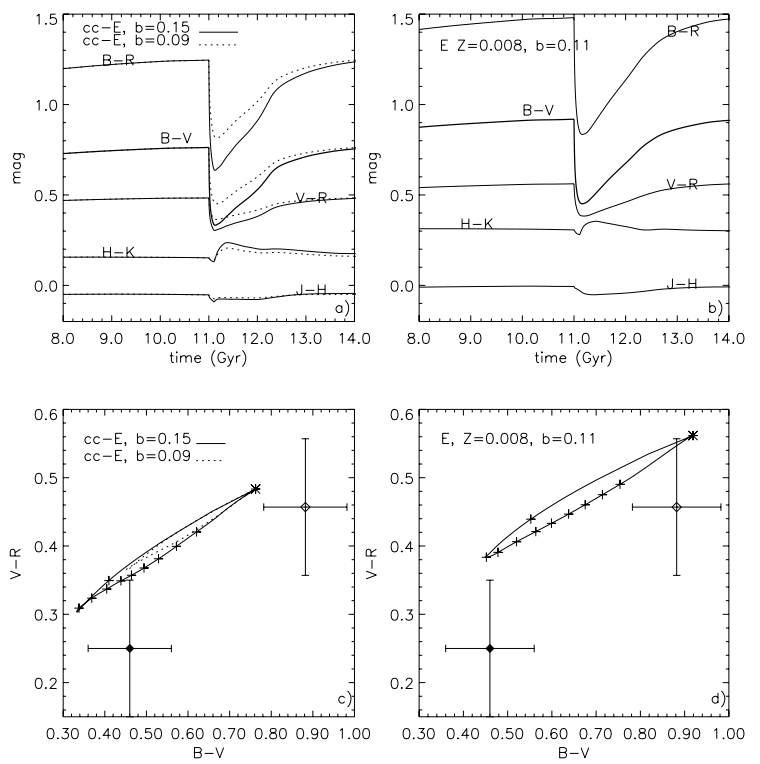

Fig. 11. a) Evolution of color indices for a CC-E model with a $15 \%$ (solid line) and a 9\% (dotted line) burst switched on after $11 \mathrm{Gyr}$ of undisturbed evolution. The $B-V$ index is indicated with a thick line to avoid confusion with other indices. For the same reason, an offset of -0.6 has been applied to the $J-H$ index. b) Same as a), for a non-CC E model with metallicity $Z=0.008$ and a $11 \%$ burst. c) Color-color diagram showing the evolution of the color indices during the burst phase for the CC-E model. Line styles are as in a). Symbols as in Fig. 8. d) Same as c) for the non-CC E model.

simulated to explain the observed SFR. Since galaxies are treated as closed boxes, we could not simulate gas accretion from the companion galaxies. The color evolution for E-type models is shown in Fig. 11. We simulated burst strengths of $27 \%, 15 \%$, and $9 \%$ with e-folding timescale of $2 \times 10^{8} \mathrm{yr}$. An analogous fitting procedure to the one described in Sect. 3 was applied, and the best value for internal extinction was found to be in the same range as for $\mathrm{Sa}$ and $\mathrm{Sb}$ models (Sect. 4.1). In all cases a good agreement between modeled and observed spectrum is reached at increasingly old burst ages for increasing burst strengths, in the range 1.3 to $1.6 \mathrm{Gyr}$ (Fig. 12), when the SFR of the model has dropped far below the observed value. Model metallicities, $\approx 0.2 Z_{\odot}$, are lower than observed, and model SEDsare not in good agreement with NIR photometry of G2 (Fig. 13), contrary to the case of Sa or Sb models. In fact a non-CC model with elliptical-like $\mathrm{SFH}$, but with metallicity of the stellar populations set to $Z=0.008$ (i.e. $0.4 Z_{\odot}$ ), gives younger burst ages and better agreement with the observed SED (Fig. 14). Such a non-CC model could be used to mimic the case in which the early-type galaxy has been accreting some relatively high-metallicity gas from the companion(s), while still keeping the number of free parameters at a minimum using a closed box model. For this last model with a burst strength of $11 \%$, the best agreement with the observed spectrum is found at burst ages 0.9-1.0 Gyr. Taking the $K$ band luminosity and the model mass-to-light ratio as references, we derived a total mass of $7 \times 10^{10} M_{\odot}$ and a stellar mass of $4 \times 10^{10} M_{\odot}$ for G2, i.e. about twice as high as derived from the Sa model. Scaling the model SFR accordingly gives $S F R \sim 0.1-0.05 M_{\odot} \mathrm{yr}^{-1}$ for 0.9 and $1.0 \mathrm{Gyr}$ burst ages, respectively. These values are lower than the measured 0.4-0.8 $M_{\odot} \mathrm{yr}^{-1}$. However, these measured values suffer from a high uncertainty in the extinction determination, because of the extreme weakness of the $\mathrm{H} \beta$ emission-line, therefore we still consider the model to be in reasonable agreement with the observations. In Table 5 we show the scaled model luminosities, masses, and SFRs and the modeled colors for different burst ages. Luminosities agree reasonably well (within the errors) with the observed ones. At ages in the range 0.9-1.0 Gyr after the onset of the burst, the newly formed stars contribute $8 \%$ of the stellar mass of the galaxy and $\sim 32 \%$ of its visual light.

\section{Multiple bursts}

The evolutionary synthesis approach, as opposed to population synthesis, does not allow unexpected SFHs to be discovered. In the models presented above, we assumed the simplest caseof a single interaction-induced burst occurring, which limits the number of free parameters. However, in the tight galaxy 

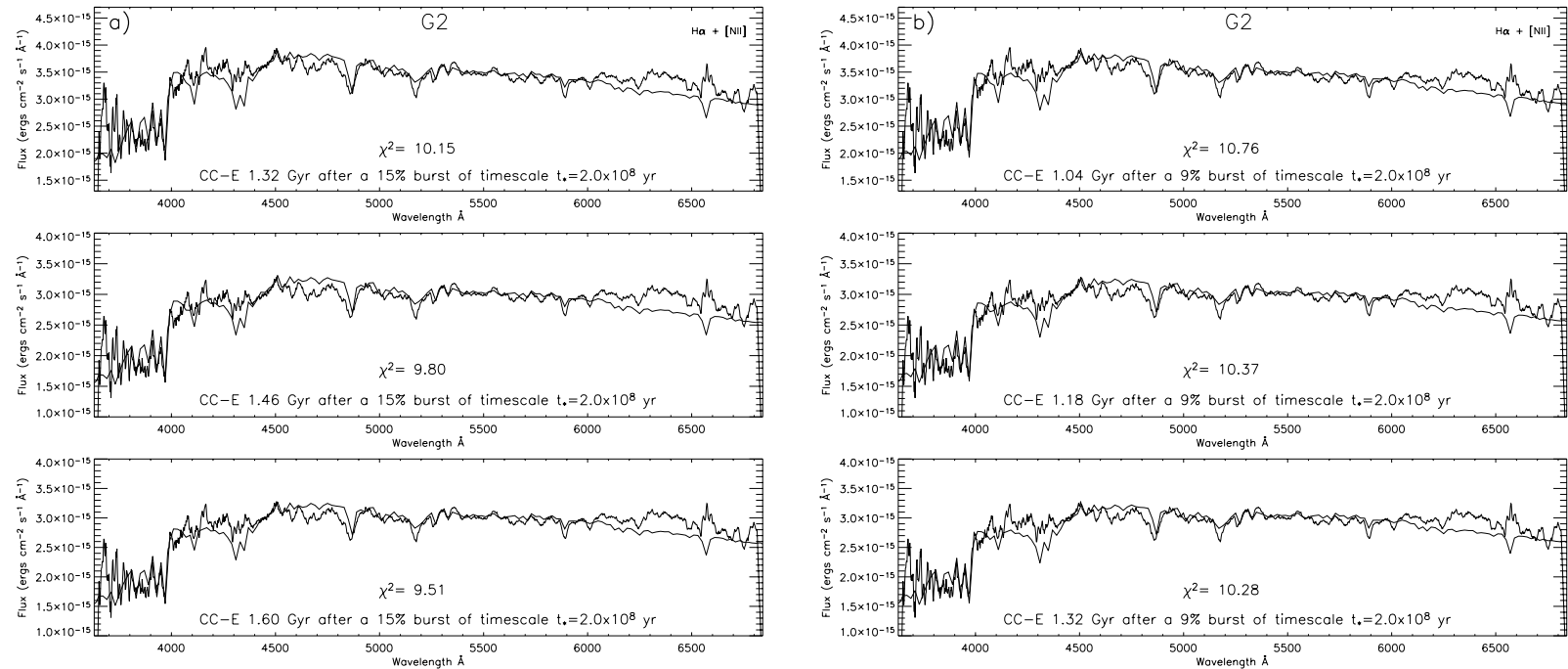

Fig. 12. a) CC-E model spectra (thick line), conveniently scaled, overplotted to the dereddened and smoothed observed spectrum of G2 at three different ages after the onset of a $15 \%$ burst with a timescale of $2 \times 10^{8} \mathrm{yr}$. The best fit occurs 1.5 to $1.6 \mathrm{Gyr}$ after the burst, for an internal extinction $E(B-V)=0.36$ mag. b) Same as a) for a $9 \%$ burst. The best fit occurs 1.3 to $1.5 \mathrm{Gyr}$ after the burst, with internal extinction $E(B-V)=0.36 \mathrm{mag}$.
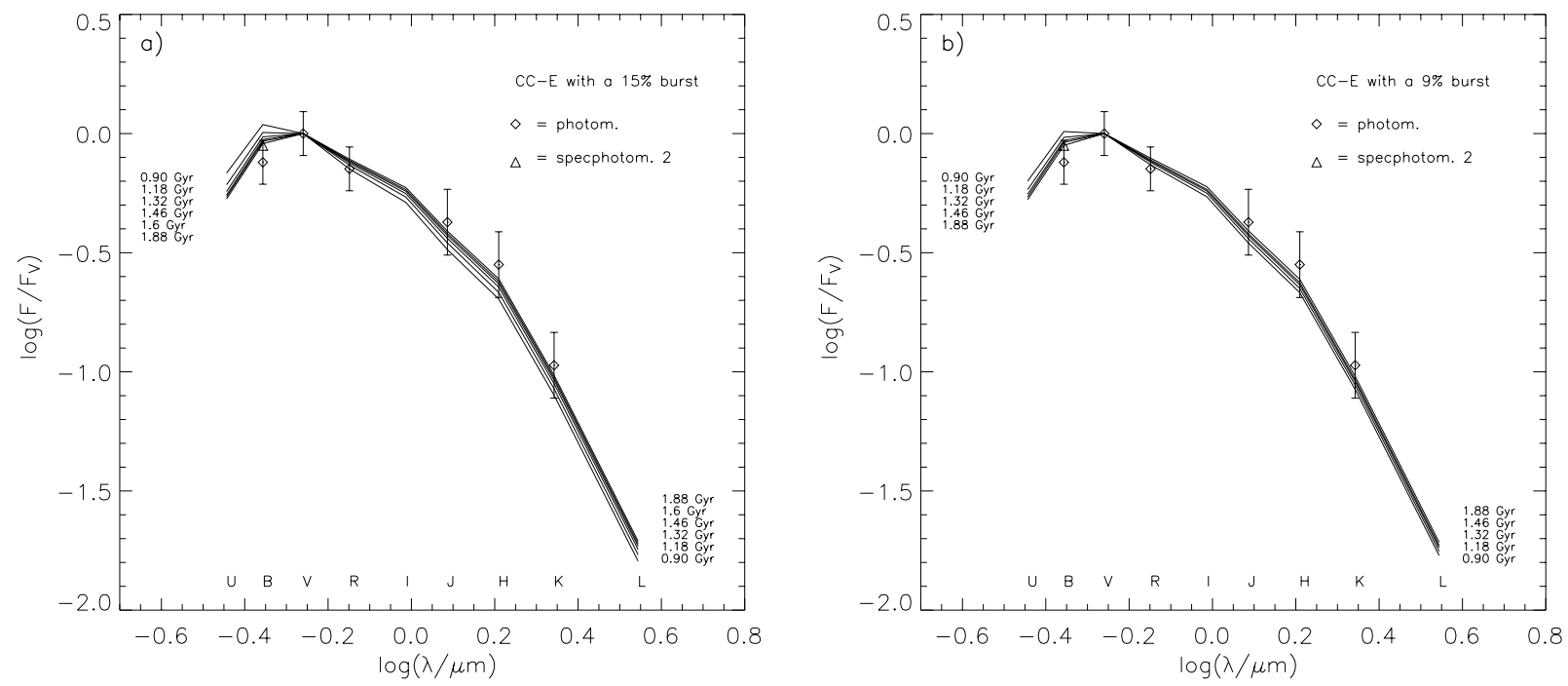

Fig. 13. SEDs for the CC-E models with burst strength 15\% (left) and 9\% (right), along with dereddened photometric (diamonds) and spectrophotometric (triangle) data points for G2. Best-fit ages according to the spectral analysis are indicated with a thick line. Discrepancies are visible in the NIR.

system under study, the galaxies may have already undergone several mutual interactions before the present one and, as a consequence, they might have experienced multiple bursts of starformation. We investigated the possibility of reproducing the observed spectrophotometric properties of the two spiral galaxies by assuming multiple bursts of star-formation. However, it turned out that the observed spectra can be reproduced only if assuming a recent burst that is much stronger than the possible previous ones. Whatever the age of previous interactioninduced bursts of star-formation, satisfactory models are obtained only 40 to $180 \mathrm{Myr}$ after the latest strong burst. Also, simulating multiple bursts implies a considerable increase in the number of free parameters, which cannot be constrained with the available observations. The consequent degeneracy in the models makes it impossible to establish the relative age and strength of possible previous bursts with any confidence. Therefore, our models do not allow us to draw any conclusions about previous bursts. We can only conclude that the spectral features are dominated by the latest strong burst. As an example, we provide the parameters of a triple-burst model here, whose match to the observational data of G4 would be as good as that of the single-burst model presented in Sect. 3. The model could also be successfully applied to G1 after rescaling to the appropriate total mass. In this model the three bursts were switched on at galaxy ages of 11.0, 11.7, and 12.0 Gyr, respectively. The burst duration was assumed to be the same, $2 \times 10^{8} \mathrm{yr}$, for each burst. Burst strengths were set to $7 \%$, $11.5 \%$, and $38 \%$, respectively. The best match to the observed spectrum occurs 40 to $180 \mathrm{Myr}$ after the latest burst, similar to the single-burst model (reduced- $\chi^{2}=3.93$ and 5.28 for 

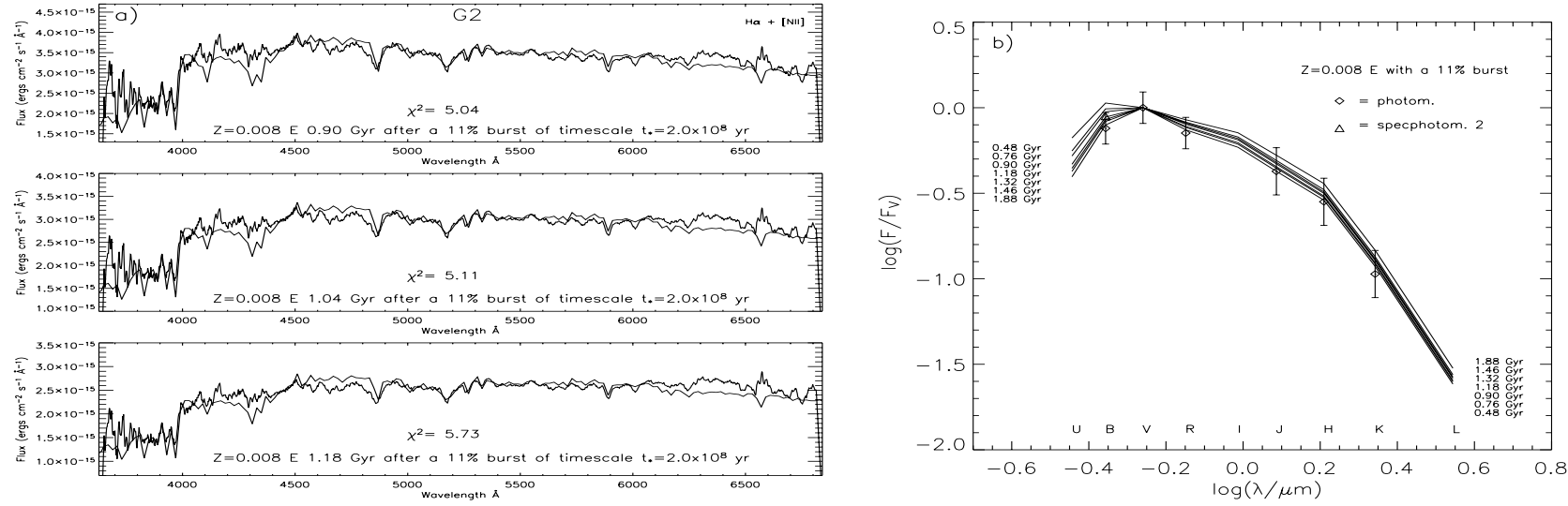

Fig. 14. a) Smoothed spectrum of G2 corrected for internal reddening and overlapped with conveniently rescaled model spectra (thick line) at three different ages after the onset of a burst on an elliptical (E) galaxy with stellar population metallicity $Z=0.008$. The best fits occurs for $E(B-V)$ in the range $0.36-0.41 \mathrm{mag}$ and burst ages of $0.90-1.04$ Gyr. b) SED for the $Z=0.008 \mathrm{E}$ model at various burst ages. Symbols as in Fig. 13.
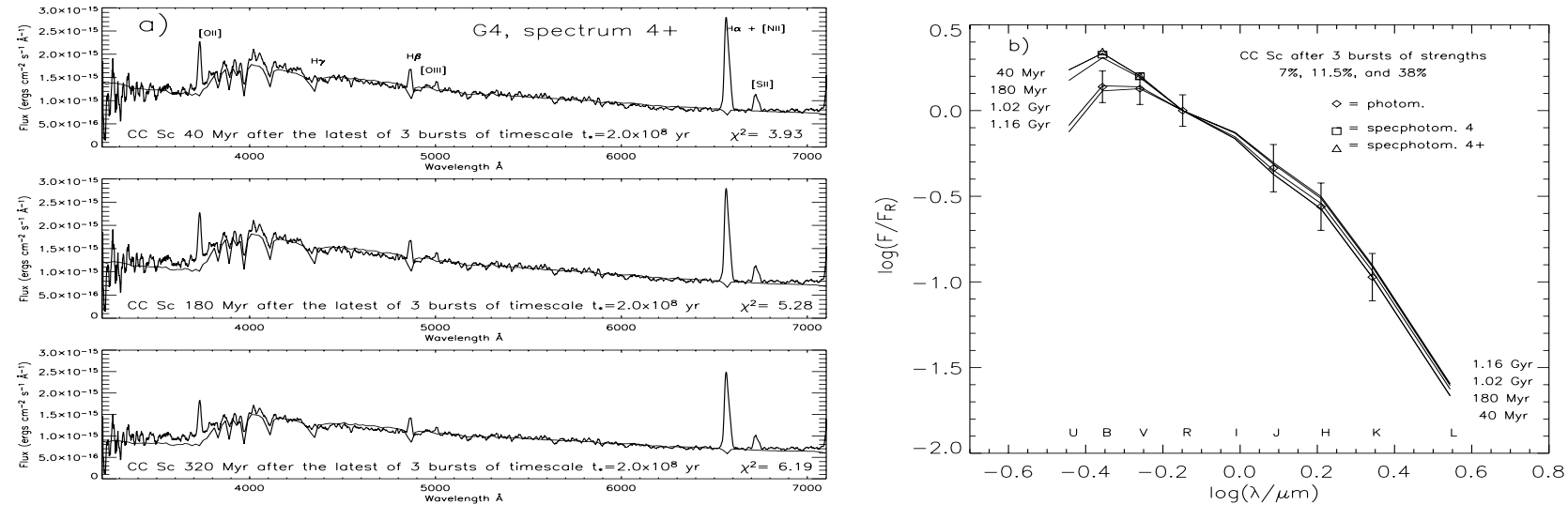

Fig. 15. a) Spectrum of G4 at PA $90^{\circ}$ corrected for internal reddening and overlapped with CC-Sc model spectra (thick line) at three different ages after the latest of three interaction-induced bursts of star-formation (see text). b) SED for the CC-Sc model at three ages after the latest of three interaction induced bursts. Photometric points of G4 are marked with diamonds, while the spectrophotometric points derived from the dereddened spectra at PA $130^{\circ}$ and $90^{\circ}$ are indicated by triangles and squares, respectively. The thick line indicates the best fit age, according to the spectral analysis.

burst ages of 40 and $180 \mathrm{Myr}$, respectively, and for a bestfit value of internal extinction $E(B-V)=0.49 \mathrm{mag}$ for the spectrum 4+). For the model $40 \mathrm{Myr}$ after the burst, the mass fractions of stars with ages $t<40 \mathrm{Myr}, 40 \mathrm{Myr} \leq t \leq$ $340 \mathrm{Myr}$, and $440 \mathrm{Myr} \leq t \leq 1 \mathrm{Gyr}$ are $\approx 7 \%, 10 \%$, and $6 \%$, respectively, with the youngest stars contributing $\approx 30 \%$ of the total $V$-band luminosity. For the model $180 \mathrm{Myr}$ after the latest burst, $\sim 20 \%$ of stars (in mass) were produced after the onset of the burst, and they contribute $\sim 47 \%$ of the light in the $V$ band. The SFRs calculated 40 and $180 \mathrm{Myr}$ after the latest burst are $\sim 12$ and $6 M_{\odot} \mathrm{yr}^{-1}$, respectively, and the relevant metallicities are 0.25 and $0.36 Z_{\odot}$. Therefore, in case of multiple burst models as well, all information taken together points to an age of the latest burst between 40 and $180 \mathrm{Myr}$. For models with previous bursts that are considerably stronger than the latest one, no agreement is found between observations and models at any age. The comparison of multiple-burst model spectra and SEDs at different ages with the observed spectroscopic and photometric data is shown in Fig. 15. The difference with respect to the single-burst models shown in
Figs. 5, 7 is only marginal. Colors, luminosities, and metallicities of the triple-burst model 40 and $180 \mathrm{Myr}$ after the latest burst are given in Table 6. Differences between colors obtained with the single-burst models and those obtained with the tripleburst model are well within the errors. Taking the model with older burst age as reference, provided the modeled mass-tolight ratio is valid for G4, the observed luminosity would imply a stellar mass of $1.5 \times 10^{10} M_{\odot}$. The accordingly scaled SFR of the model would be $\sim 5.5 M_{\odot} \mathrm{yr}^{-1}$, still in reasonable agreement with the observations, while the metallicity reached by the model is higher than observed. Slightly higher total and stellar masses $\left(\sim 2.4 \times 10^{10} M_{\odot}\right.$ and $\sim 1.8 \times 10^{10} M_{\odot}$, respectively) would be implied by the 40 Myr old burst model, but the model SFR would be too high with respect to the observations $\left(\sim 14.9 M_{\odot} \mathrm{yr}^{-1}\right)$.

\section{Discussion and conclusions}

We have used the Göttingen evolutionary synthesis code GALEV to obtain chemically consistent spectrophotometric 
Table 6. G4 - Observed ${ }^{a}$ and triple-burst model colors and luminosities.

\begin{tabular}{llllllll}
\hline \hline Object/Model & $B-V$ & $V-R$ & $V-K$ & $B-R$ & $B-H$ & $J-H$ & $H-K$ \\
& $M_{B}$ & $M_{V}$ & $M_{R}$ & $M_{J}$ & $M_{H}$ & $M_{K}$ & $Z / Z_{\odot}$ \\
\hline G4 & 0.55 & 0.51 & 2.20 & 1.06 & 2.57 & 0.48 & 0.18 \\
& -20.30 & -20.86 & -21.36 & -22.40 & -22.87 & -23.06 & $\sim 0.1$ \\
CC-Sc, 3 bursts, $40 \mathrm{Myr}$ & 0.26 & 0.34 & 1.98 & 0.61 & 2.04 & 0.52 & 0.20 \\
$M=2.38 \times 10^{10} M_{\odot}$ & -20.81 & -21.08 & -21.41 & -22.34 & -22.85 & -23.06 & 0.25 \\
CC-Sc, 3 bursts, $180 \mathrm{Myr}$ & 0.25 & 0.32 & 2.01 & 0.57 & 2.05 & 0.53 & 0.22 \\
$M=1.77 \times 10^{10} M_{\odot}$ & -20.80 & -21.05 & -21.37 & -22.32 & -22.85 & -23.06 & 0.36 \\
\hline
\end{tabular}

${ }^{a}$ Observed luminosities and colors after the application of a standard inclination correction.

evolutionary synthesis models to be compared to the three strongly interacting, star-forming galaxies in the compact group CG J1720-67.8.

Our goal was to find the simplest solutions that account for the observed properties of the galaxies within the observational and model uncertainties. The models we have presented and compared with the observational data were obtained under a number of simplifying assumptions, such as considering each galaxy as a closed box and assuming an undisturbed evolution - with a SFH consistent with the morphological type of the galaxies - before the present/last encounter/starburst event. These assumptions are justified by the need to keep the number of free parameters as small as possible, because of the impossibility of constraining a larger number of parameters with the limited amount of data at our disposal. As a result, the accuracy of the solutions presented here is necessarily limited, and some degree of degeneracy between the model parameters (e.g. progenitor type and/or actual value of internal extinction and burst strength) still remains.

The interaction history of CG1720-67.8 probably involves multiple encounters and induced bursts of star-formation. Therefore, we have investigated the possibility of multiple bursts occurring in the spiral galaxies. However, no unique solution can be found with the presently available data, because of the high number of additional free parameters involved, such as the duration of the individual bursts, their number and time separation, and their relative strengths.

We provided an example of a multiple-burst model that would give as good a solution as the simpler single-burst model applied to G4. Although we cannot establish how many interaction-induced bursts of star-formation have occurred in the galaxy's history, our models indicate that the presently observed properties are reproduced best when the latest burst episode is assumed to be stronger than previous bursts. This result is consistent with the widely accepted belief that major bursts destroy galaxy disks, which at best can be rebuilt on a timescale of 3-4 Gyr as small, Sa-type disks. Since galaxies G1 and G4 both still show significant disks, we can rule out the possibility that they have undergone particularly strong bursts in the last $3 \mathrm{Gyr}$, before the presently observed one. A set of data spanning a wider wavelength range from UV to NIR, as well as a dynamical model of the entire galaxy group would provide constraints for additional parameters, thus giving a basis for more sophisticated evolutionary synthesis models, which could include mass transfer or exchange either between galaxies or with the intragroup medium, besides multiple bursts. Work to build a dynamical model of the galaxy group is presently in progress. A further limitation to our models is given by the lack of an accurate knowledge of the internal extinction, as discussed in Sects. 3 and 4. However, with the above caveats in mind and taking the uncertainties into account (both in the observations and the models), we were able to obtain models that are reasonably consistent with the observational data. These give us an indication of the age and strength of the (latest) interaction-induced star-formation episodes in the galaxies and yield estimates of the stellar masses of the galaxies.

In the version of GALEV we used, the contribution of gaseous emission to broadband fluxes was not included. However, the models presented here have burst ages $\geq 40 \mathrm{Myr}$. At these ages the contribution of ionized gas emission (both in terms of lines and continuum) to broadband fluxes is negligible at any metallicity, as shown by Anders \& Fritze-v. Alvensleben (2003).

We found that the two late-type group's spirals, G1 and G4, have SEDs and optical spectra in reasonable agreement with a CC-model of an Sc galaxy at an age $\sim 40-180$ Myr after the onset of an interaction-induced intermediate to strong burst of star-formation. In particular, the strong older burst offers the preferred solution for G1, while intermediate ages and strengths between the two proposed models might be more suitable to G4. From the $K$-band luminosity of G4 and the mass-to-light ratio of the preferred model, we derived a stellar mass of $\sim 1.6 \times 10^{10} M_{\odot}$ and a total (stellar + gaseous) mass of $\approx 2 \times 10^{10} M_{\odot}$. This luminous mass estimate appears reasonable when compared with the dynamical mass of $2.2 \times 10^{10} M_{\odot}$ derived in Paper IV from the rotation curve of the galaxy within a radius of $5^{\prime \prime}$, nearly one disk-scale-length. Using the fitting law to the rotation curve and extrapolating to a radius of $8^{\prime \prime}$ (i.e. nearly two disk-scale-lengths) would give a dynamical mass of $3.3 \times 10^{10} M_{\odot}$. For G1 we took the $R$-band luminosity as reference, because measured NIR luminosities are contaminated by an overlapping, foreground M-type star, and $B V$ luminosities are more affected by extinction. The estimated total mass of G1 is in the range 4 to $6 \times 10^{9} M_{\odot}$. We estimated that the burst increased the stellar mass of these galaxies by 9 to 24 per cent. 
For the early-type galaxy G2, we found two alternative solutions that lead to significantly different estimates of the galaxy's total mass. The first model explores the possibility that the galaxy results from the merging of two gas-poor early-type spirals, an idea that was suggested in Paper IV on the basis of the observational properties of the group, including its tidal features. Data appear consistent with a model in which two Sa galaxies, each one $\sim 1.7 \times 10^{10} M_{\odot}$ in mass, experienced a strong - as far as their gas reservoir allowed - burst of star-formation during their merging process $\sim 0.7-0.9 \mathrm{Gyr}$ ago, thereby increasing the stellar mass of the galaxy by $\sim 5 \%$. However, two $\mathrm{Sb}$ progenitors of $\sim 1.4 \times 10^{10} M_{\odot}$ each would also offer an acceptable solution and would imply a burst age of $\sim 1.2-1.3 \mathrm{Gyr}$ and a stellar mass increase of $\sim 11 \%$.

The alternative model considers the possibility that $\mathrm{G} 2$ was born as an early-type galaxy and that it recently experienced a central burst of star-formation because of gas inflow induced by the interaction with its companions. A solution that is reasonably consistent with the data was found using a $Z=0.44 Z_{\odot}$ model with E-type $\mathrm{SFH}$, and it indicates a burst age in the range $0.9-1.0 \mathrm{Gyr}$, with a contributed stellar mass of $8 \%$. The total mass derived for $\mathrm{G} 2$ is $3.4 \times 10^{10} M_{\odot}$ for the CC-Sa merger model and $7 \times 10^{10} M_{\odot}$ for the non-CC E model.

The burst ages we found for the two spiral galaxies are largely consistent with our rough estimate of the age of the long tidal tail responsible for $\sim 30 \%$ of the group's optical luminosity. This supports the idea that the tail was issued as a consequence of a recent interaction between the two spirals. Although it is not possible to establish whether the S0 galaxy was formed through a late merger event or was born as an early-type galaxy, the models seem to indicate that the episode of star-formation, whose traces are still present in the galaxy spectrum, is relatively old compared to the burst age of the two companion galaxies. This suggests that G2 is not involved in the interaction that produced the long tidal tail, but might be responsible for those fainter tidal features visible in the group that can be interpreted as fading remnants of older interaction processes. Detailed dynamical models should further help in disentangling the complex interaction history of this galaxy group. The total (stellar plus gaseous) mass of the galaxies involved is $z 6 \times 10^{10} M_{\odot}$, without considering the material contained in the main tidal tail, whose contribution is nonnegligible, judging from its percentage luminosity. Therefore, the visible mass of the final group remnant is expected to be on order of $\lesssim 10^{11} M_{\odot}$.

In the following, we discuss our results in a more general context by comparing them to published works on interacting galaxies and/or small galaxy groups. Although following a different approach from the one we adopted in this work, Barton Gillespie et al. (2003) estimate the ages, strengths, and timescales of bursts of induced star-formation at the center of galaxies in pairs. They find indication that the bursts in the galaxies with small separations and large $\mathrm{H} \alpha$ equivalent widths are typically younger and stronger than in other galaxies. They conclude that the strengths and ages of triggered starbursts probably depend on galaxy separation on the sky and suggest that low-mass galaxies experience stronger bursts of triggered star-formation. Their observations appear consistent with bursts of star-formation that are switched on at a close galaxy-galaxy pass and that continue and age while the galaxies move apart. The results we presented in this work for CG J1720-67.8 appear consistent with the scenario depicted by Barton Gillespie et al. (2003).

We probed processes at work in a very compact galaxy configuration found in isolation, which we believe is approaching coalescence. It is unclear whether a similar behavior is to be expected for analogously compact configurations embedded in denser environments. Recent investigations by Coziol et al. (2004) suggest that in such cases group evolution might proceed from low-velocity dispersion groups with active star-formation, through intermediate-velocity dispersion groups containing a large fraction of interacting/merging galaxies, and then to high-velocity dispersion groups dominated by inactive elliptical galaxies. To clear up this point, detailed studies would be particularly useful, similar to the one on CG J1720-67.8 that we presented in this series of papers, but targeting very compact galaxy systems embedded in environments with differing densities.

In a recent study, Balogh et al. (2004) analyze the dependence of star-formation activity on environmental density by considering group catalogs extracted from the $2 \mathrm{dF}$ Galaxy Redshift Survey (2dFGRS, Colless et al. 2001) and the Sloan Digital Sky Survey (SDSS, Abazajian et al. 2003). They find that the fraction of star-forming galaxies depends strongly on local density and also on large-scale density, approximately independent of group velocity dispersion, while the distribution of $\mathrm{H} \alpha$ equivalent width in the star-forming population does not depend on the environment. However, their analysis excludes the particular category/evolutionary phase of groups, of which CG J1720-67.4 is an example. In fact their study is limited to groups with velocity dispersion $>200 \mathrm{~km} \mathrm{~s}^{-1}$ and with at least 10 member galaxies. Additionally, extremely compact galaxy configurations, like the one considered in our work, cannot be selected in redshift space from the SDSS and 2dFGRS, because of observational constraints that impose a lower limit to the projected angular separation between galaxies for which spectra can be obtained. At lower velocity dispersions and small galaxy separations, interaction processes are more effective and induced star-formation can become important. It is this situation that is expected to dominate the coalescence phase in group evolution, i.e. the phase leading to the formation of "fossil" groups. In fact, in our work we have shown that the star-formation activity of the galaxies in the chosen group is strongly related to their recent interaction history. Moderate to strong bursts of star-formation have been switched on in two of the three members at an age that is compatible with the time of the latest strong interaction, namely within the last $200 \mathrm{Myr}$, as indicated by the length of the tidal tail. An older $(\approx 1 \mathrm{Gyr})$, not yet fully extinguished, star-formation episode located in the early-type galaxy suggests an older interaction or even the first merger event in the history of the group. As already discussed in Paper IV, the global star-formation activity of CG J1720-67.8 is more intense, by at least a factor of 4 , than that displayed by several well-studied merging galaxy pairs. As a further term of comparison, we can consider the triple galaxy system AM 1238-362, whose properties have 
been studied in detail by Temporin et al. (2003c). This last system is also characterized by very low velocity dispersion, but its median projected galaxy separation $(\sim 35 \mathrm{kpc})$ is larger than that of CG J1720-67.8, and its members are all spiral galaxies, one of which hosts a Seyfert 2 nucleus. Although morphological distortions in this small group are weak, at least two of its members show enhanced star-formation activity, which has been suggested as the effect of a recent close passage in their orbits. Several candidate intergalactic H II regions, another possible consequence of the interaction, have been identified in the immediate vicinity of these galaxies (Temporin et al. 2005b). Star-formation, although more intense in the central regions of the galaxies, is spread across their disks, analogous to what we observed in the spiral members of CG J1720-67.8. The SFR surface densities derived for the more active member of the triplet, Tol 1238-364, from $\mathrm{H} \alpha$ line emission measurements are comparable with those derived for G1 and G4 in Paper II. With respect to AM 1238-362, CG J1720-67.8 appears to represent a later evolutionary phase in which galaxies have come closer and are undergoing more violent interactions.

By studying a subsample of compact groups from the Hickson (1982) catalog, Iglesias-Páramo \& Vílchez (1999) found no global enhancement of SFR in compact group members with respect to a sample of field galaxies. However, for a few groups, they found a ratio of $\mathrm{H} \alpha$ to $B$ luminosity that is significantly higher than values measured in synthetic groups built from their field galaxy sample. Most of these groups contain interacting galaxy pairs with evident tidal features. One of these is the well-studied group, HCG 31, which is believed to have started its merging phase and shows similarities with CG J1720-67.8 concerning its peculiar morphology, dynamical properties, star-formation activity, and the presence of candidate tidal dwarf galaxies (Amram et al. 2004; López-Sánchez et al. 2004; Mendes de Oliveira et al. 2005b). The star-formation history of HCG 31 has been recently investigated by López-Sánchez et al. (2004), who confirmed the previous claims of very young ages of the latest bursts of star-formation, mostly $<10 \mathrm{Myr}$, for the members of this group. Considering our burst age estimates for the galaxies of CG J1720-67.8 and for its tidal dwarf galaxy candidates ( $<10 \mathrm{Myr}$, Paper IV), we suggest that the group is observed at a similar (or slightly later) phase to HCG 31 in the merging process.

If CG J1720-67.8 is to be considered as representative of a late pre-merging phase in poor groups, its properties suggest that sufficiently gas-rich groups should undergo a particularly active star-forming phase before final coalescence.

Acknowledgements. This work was supported by the Austrian Science Fund (FWF) under projects P15065 and P17772. S.T. gratefully acknowledges hospitality at the Universitätssternwarte Göttingen and travel support from the German Astronomische Gesellschaft. We are grateful to the anonymous referee for the useful and constructive comments, which helped improve the presentation of our work. S.T. is grateful to J. Fritz and S. Ciroi for helpful suggestions and discussions.

\section{References}

Abazajian, K., Adelman-McCarthy, J. K., Agüeros, M. A., et al. 2003, AJ, 126, 2081

Anders, P., \& Fritze-v. Alvensleben, U. 2003, A\&A, 401, 1063

Amram, P., Mendes de Oliveira, C., Plana, H., et al. 2004, ApJ, 612, L5

Athanassoula, E., Makino, J., \& Bosma, A. 1997, MNRAS, 286, 825

Balogh, M., Eke, V., Miller, C., et al. 2004, MNRAS, 348, 1355

Barnes, J. E., \& Hernquist, L. 1991, ApJ, 370, L65

Barnes, J. E., \& Hernquist, L. 1996, ApJ, 471, 115

Barton Gillespie, E., Geller, M. J., \& Kenyon, S. J. 2003, ApJ, 582, 668

Bertelli, G., Bressan, A., Chiosi, C., Fagotto, F., \& Nasi, E. 1994, A\&AS, 106, 275

Bessell, M. S. 1990, PASP, 102, 1881

Bicker, J., Fritze-v. Alvensleben, U., Möller, C. S., \& Fricke, K. J. 2004, A\&A, 413, 37

Buta, R., Corwin, H. G. Jr., de Vaucouleurs, G., de Vaucouleurs, A., \& Longo, G. 1995, AJ, 109, 517

Buta, R., \& Williams, K. L. 1995, AJ, 109, 543

Calzetti, D. 2001, PASP, 113, 1449

Calzetti, D., Kinney, A. L., \& Storchi-Bergman, T. 1994, ApJ, 429, 582

Cardelli, J. A., Clayton, G. C., \& Mathis, J. S. 1989, ApJ, 345, 245

Chabrier, G., \& Baraffe, I. 1997, A\&A, 327, 1039

Colless, M., Dalton, G., Maddox, S., et al. 2001, MNRAS, 328, 1039

Cole, S., Lacey, C. G., Baugh, C. M., \& Frenk, C. S. 2000, MNRAS, 319, 168

Coziol, R., Brinks, E., \& Bravo-Alfaro, H. 2004, AJ, 128, 68

Ferland, G. J. 1996, HAZY, a brief introduction to Cloudy, University of Kentucky, Department of Physics and Astronomy Internal Report

Fritze-v. Alvensleben, U. 1999, ASP Conf. Ser., 192, 273

Fritze-v. Alvensleben, U., \& Gerhard, O. E. 1994, A\&A, 285, 751

Hickson, P. 1982, ApJ, 255, 382

Iglesias-Páramo, J., \& Vílchez, J. M. 1999, ApJ, 518, 94

Kauffmann, G., Colberg, J., Diaferio, A., \& White, S. D. M. 1999, MNRAS, 303, 188

Kennicutt Jr., R. C. 1992, ApJS, 79, 254

Kennicutt Jr., R. C. 1998a, ApJ, 498, 541

Kennicutt Jr., R. C. 1998b, ARA\&A, 36, 189

Krüger, H., Fritze-v. Alvensleben, U., \& Loose, H.-H. 1995, A\&A, 303,41

Kurth, O. M., Fritze-v. Alvensleben, U., \& Fricke, K. J. 1999, A\&AS, 138, 19

Lacey, C., \& Cole, S. 1993, MNRAS, 262, 627

Lejeune, Th., Cuisinier, F., \& Buser, R. 1997, A\&AS, 125, 229

Lejeune, Th., Cuisinier, F., \& Buser, R. 1998, A\&AS, 130, 65

Leonardi, A. J., \& Rose, J. A. 1996, AJ, 111, 182

Lindner, U., Fritze-von Alvensleben, U., \& Fricke, K. J. 1999, A\&A, 341, 709

López-Sánchez, A., Esteban, C., \& Rodríguez, M. 2004, ApJS, 153, 243

Mendes de Oliveira, C., Coelho, P., González, J. J., \& Barbuy, B. 2005a, AJ, 130, 55

Mendes de Oliveira, C., Temporin, S., Cypriano, E., et al. 2005b, AJ, submitted

Miles, T. A., Raychaudhury, S., Forbes, D. A., et al. 2004, MNRAS, 355,785

Mulchaey, J. S., \& Zabludoff, A. I. 1999, ApJ, 514, 133

Mihos, J. C., Bothun, G. D., \& Richstone, D. O. 1993, ApJ, 418, 82

Mihos, J. C., \& Hernquist, L. 1994, ApJ, 431, L9 
Noguchi, M. 1988, A\&A, 203, 259

Ponman, T. J., Allan, D. J., Jones, L. R., et al. 1994, Nature, 369, 462 Proctor, R. N., Forbes, D. A., Hau, G. K. T., et al. 2004, MNRAS, 349, 1381

Rose, J. A. 1984, AJ, 89, 1238

Salpeter, E. E. 1955, ApJ, 121, 161

Sandage, A., Binggeli, B., \& Tammann, G. A. 1985, AJ, 90, 1759

Scalo, J. M. 1986, Fund. Cosmic Phys., 11, 1

Schulz, J., Fritze-v. Alvensleben, U., Möller, C. S., \& Fricke, K. J. 2002, A\&A, 392, 1

Springel, V., White, S. D., Jenkins, A., et al. 2005, Nature, 435, 629

Tinsley, B. M. 1980, Fund. Cosmic Phys., 5, 287

Temporin, S., Weinberger, R., Galaz, G., \& Kerber, F. 2003a, ApJ, 584, 239 (Paper II)
Temporin, S., Weinberger, R., Galaz, G., \& Kerber, F. 2003b, ApJ, 587, 660 (Paper III)

Temporin, S., Ciroi, S., Rafanelli, P., et al. 2003, ApJS, 148, 353

Temporin, S., Staveley-Smith, L., \& Kerber, F. 2005a, MNRAS, 356, 343 (Paper IV)

Temporin, S., Ciroi, S., Iovino, A., et al. 2005b, in Starbursts: From 30 Doradus to Lyman Break Galaxies, ed. R. de Grijs, \& R. M. González Delgado (Dordrecht: Springer), Ap\&SS, Library, 329, P78

Vikhlinin, A., McNamara, B. R., Hornstrup, A., et al. 1999, ApJ, 520, L1

Weinberger, R., Temporin, S., \& Kerber F. 1999, ApJ, 522, L17 (Paper I)

Worthey, G. 1994, ApJS, 95, 107 